\title{
Exact Peakon, Compacton, Solitary Wave, and Periodic Wave Solutions for a Generalized KdV Equation
}

\author{
Qing Meng ${ }^{1}$ and Bin $\mathrm{He}^{2}$ \\ ${ }^{1}$ Department of Physics, Honghe University, Mengzi, Yunnan 661100, China \\ ${ }^{2}$ College of Mathematics, Honghe University, Mengzi, Yunnan 661100, China \\ Correspondence should be addressed to Bin He; hebinmtc@163.com
}

Received 29 August 2013; Accepted 25 September 2013

Academic Editor: Jun-Juh Yan

Copyright (C) 2013 Q. Meng and B. He. This is an open access article distributed under the Creative Commons Attribution License, which permits unrestricted use, distribution, and reproduction in any medium, provided the original work is properly cited.

\begin{abstract}
We employ the approaches of both dynamical system and numerical simulation to investigate a generalized KdV equation, which is presented by Yin (2012). Some peakon, compacton, solitary wave, smooth periodic wave, and periodic cusp wave solutions are obtained, and the planar graphs of the compactons and the periodic cusp waves are simulated.
\end{abstract}

\section{Introduction}

To study the role of nonlinear dispersion in the formation of patterns in the liquid drop, Rosenau and Hyman [1] showed in a particular generalization of the $\mathrm{KdV}$ equation

$$
u_{t}+\left(u^{2}\right)_{x}+\left(u^{2}\right)_{x x x}=0
$$

which is called $K(2,2)$ equation. They found some solitary waves with compact support in it, which they called compactons. These compactons had the property that the width was independent of the amplitude. Equation (1) has been studied successfully by some authors [2-8]. However, (1) does not exhibit the usual energy conservation law. Instead of (1), Cooper et al. considered the corresponding generalized $\mathrm{KdV}$ equation [9]

$$
u_{t}+u u_{x}+\alpha\left(2 u u_{x x x}+4 u_{x} u_{x x}\right)=0,
$$

which can be derived from a Lagrangian. Equation (2) has the same terms as (1), except the relative weights of the terms. Equation (2) also admits compacton solutions. The stability of the compacton solutions to (2) was studied in [10]. In the presence of a linear dispersion term, (2) turns into a generalized $\mathrm{KdV}$ equation with combined dispersion [11]

$$
u_{t}+u u_{x}+\alpha\left(2 u u_{x x x}+4 u_{x} u_{x x}\right)+\beta u_{x x x}=0
$$

Obviously, for $\beta=0$, (3) turns into (2). Yin [11] indicated that (3) has two conservative laws and showed that the smooth solitary waves are stable for any speed of wave propagation.

In this paper, we investigated (3) using the approaches of dynamical system and numerical simulation [12-14], we will give some new exact travelling wave solutions and simulate the compactons and the periodic cusp waves.

Next, we always suppose that $\alpha \neq 0$. Using the following independent variable transformation:

$$
u(x, t)=\phi(\xi), \quad \xi=x-c t,
$$

where $c(c \neq 0)$ is the wave speed, and substituting (4) into (3), we obtain

$$
-c \phi^{\prime}+\phi \phi^{\prime}+\alpha\left(2 \phi \phi^{\prime \prime \prime}+4 \phi^{\prime} \phi^{\prime \prime}\right)+\beta \phi^{\prime \prime \prime}=0,
$$

where " " is the derivative with respect to $\xi$.

Integrating (5) once with respect to $\xi$, we have

$$
-c \phi+\frac{1}{2} \phi^{2}+\alpha\left(2 \phi \phi^{\prime \prime}+\left(\phi^{\prime}\right)^{2}\right)+\beta \phi^{\prime \prime}=g,
$$

where $g$ is the integral constant.

Letting $y=d \phi / d \xi$, we get the following planar dynamical system:

$$
\frac{d \phi}{d \xi}=y, \quad \frac{d y}{d \xi}=\frac{g+c \phi-(1 / 2) \phi^{2}-\alpha y^{2}}{2 \alpha \phi+\beta} .
$$


The rest of this paper is organized as follows. In Section 2, we discuss the bifurcation sets and phase portraits of system (7), where explicit parametric conditions will be derived. In Section 3, we give some exact travelling wave solutions which include peakon, compacton, solitary wave, smooth periodic wave, and periodic cusp wave solutions of (3). In Section 4 , the numerical simulations of the compactons and the periodic cusp waves are given. A short conclusion will be given in Section 5.

\section{Bifurcation Sets and Phase Portraits of System (7)}

Using the transformation $d \xi=(2 \alpha \phi+\beta) d \tau$, it carries (7) into the Hamiltonian system

$$
\frac{d \phi}{d \tau}=(2 \alpha \phi+\beta) y, \quad \frac{d y}{d \tau}=g+c \phi-\frac{1}{2} \phi^{2}-\alpha y^{2}
$$

System (8) has the following first integral:

$$
H(\phi, y)=(2 \alpha \phi+\beta) y^{2}-\left(2 g \phi+c \phi^{2}-\frac{1}{3} \phi^{3}\right)=h .
$$

For a fixed $h$, the level curve $H(\phi, y)=h$ defined by (9) determines a set of invariant curves of system (8) which contains different branches of curves. As $h$ is varied, it defines different families of orbits of (8) with different dynamical behaviors.

Write $\Delta=-2 \alpha\left(\beta^{2}+4 \alpha \beta c-8 g \alpha^{2}\right), \phi_{s}=-\beta / 2 \alpha$. Clearly, when $g>-c^{2} / 2$, system (8) has two equilibrium points at $\left(\phi_{1}, 0\right)$ and $\left(\phi_{2}, 0\right)$ in $\phi$-axis, where $\phi_{1,2}=c \pm \sqrt{2 g+c^{2}}$. When $g=-c^{2} / 2$, system (8) has only one equilibrium point at $(c, 0)$ in $\phi$-axis. When $\Delta>0$, there exist two equilibrium points of system (8) in line $\phi=\phi_{s}$ at $\left(\phi_{s}, Y_{ \pm}\right)$, where $Y_{ \pm}= \pm\left(1 / 4 \alpha^{2}\right) \sqrt{\Delta}$. When $\Delta<0$, there is no equilibrium point of system (8) in line $\phi=\phi_{s}$.

Let $M\left(\phi_{e}, y_{e}\right)$ be the coefficient matrix of the linearized system of (8) at equilibrium point $\left(\phi_{e}, y_{e}\right)$; then we have Trace $M\left(\phi_{e}, 0\right)=0$ and

$$
\begin{aligned}
& J\left(\phi_{1}, 0\right)=\operatorname{det} M\left(\phi_{1}, 0\right) \\
&=\sqrt{2 g+c^{2}}\left(2 \alpha \sqrt{2 g+c^{2}}+(2 \alpha c+\beta)\right), \\
& J\left(\phi_{2}, 0\right)=\operatorname{det} M\left(\phi_{2}, 0\right) \\
&=\sqrt{2 g+c^{2}}\left(2 \alpha \sqrt{2 g+c^{2}}-(2 \alpha c+\beta)\right), \\
& J\left(\phi_{s}, Y_{ \pm}\right)=\operatorname{det} M\left(\phi_{s}, Y_{ \pm}\right)=-4 \alpha^{2} Y_{ \pm}^{2} .
\end{aligned}
$$

For an equilibrium point $\left(\phi_{e}, y_{e}\right)$ of a planar integrable system, we know that $\left(\phi_{e}, y_{e}\right)$ is a saddle point if $J\left(\phi_{e}, y_{e}\right)<0$, a center point if $J\left(\phi_{e}, y_{e}\right)>0$ and Trace $M\left(\phi_{e}, y_{e}\right)=0$, and a cusp if $J\left(\phi_{e}, y_{e}\right)=0$ and the Poincaré index of $\left(\phi_{e}, y_{e}\right)$ is zero.

Since both systems (7) and (8) have the same first integral (9), then two systems above have the same topological phase portraits except the line $\phi=\phi_{s}$. Therefore we can obtain the bifurcation sets and phase portraits of system (7) from those of system (8).

By using the properties of equilibrium points and bifurcation method of dynamical systems, we can show that bifurcation sets and phase portraits of system (7) are drawn in Figures 1 and 2.

\section{Exact Travelling Wave Solutions of (3)}

Denote that $h_{1,2}=-(2 c / 3)\left(3 g+c^{2}\right) \mp(2 / 3)\left(2 g+c^{2}\right) \sqrt{2 g+c^{2}}$ and $h_{s}=-\beta\left(\beta^{2}+6 \alpha \beta c-24 g \alpha^{2}\right) / 24 \alpha^{3}$; we present some exact travelling wave solutions of (3) as follows.

3.1. Peakon Solutions. From Figure 2(d), we see that there are two heteroclinic orbits of system (7) defined by $H(\phi, y)=$ $-\beta\left(\beta^{2}+12 \alpha \beta c+36 \alpha^{2} c^{2}\right) / 96 \alpha^{3}$ connecting with the saddle points $\left(\phi_{s}, \pm\left((2 \alpha c+\beta) \sqrt{-6 \alpha} / 8 \alpha^{2}\right)\right)$ and $((6 \alpha c+\beta) / 4 \alpha, 0)$ when $\alpha<0, c<-\beta / 2 \alpha$, and $g=-\left(12 \alpha^{2} c^{2}-4 \alpha \beta c-\beta^{2}\right) / 32 \alpha^{2}$. Their expressions are

$$
y= \pm \frac{1}{\sqrt{-6 \alpha}}\left(\phi-\frac{6 \alpha c+\beta}{4 \alpha}\right), \quad \frac{6 \alpha c+\beta}{4 \alpha}<\phi \leq \phi_{s} .
$$

Substituting (11) into $d \phi / d \xi=y$ and integrating it along the heteroclinic orbits yield equation

$$
\int_{\phi}^{\phi_{s}} \frac{d s}{s-((6 \alpha c+\beta) / 4 \alpha)}=\frac{1}{\sqrt{-6 \alpha}} \int_{0}^{\xi} d s .
$$

Completing (12) and using transformation (4), we can get a peakon solution of (3) as follow:

$$
u(x, t)=\frac{6 \alpha c+\beta}{4 \alpha}+\left(\phi_{s}-\frac{6 \alpha c+\beta}{4 \alpha}\right) e^{-\omega|x-c t|},
$$

where $\omega=1 / \sqrt{-6 \alpha}$. The profiles of (13) are shown in Figures 3(a) and 3(b).

From Figure $2(\mathrm{k})$, we see that there are two heteroclinic orbits of system (7) defined by $H(\phi, y)=-\beta\left(\beta^{2}+12 \alpha \beta c+\right.$ $\left.36 \alpha^{2} c^{2}\right) / 96 \alpha^{3}$ connecting with the saddle points $\left(\phi_{s}, \mp((2 \alpha c+\right.$ $\left.\left.\beta) \sqrt{-6 \alpha} / 8 \alpha^{2}\right)\right)$ and $((6 \alpha c+\beta) / 4 \alpha, 0)$ when $\alpha<0, c>-\beta / 2 \alpha$, and $g=-\left(12 \alpha^{2} c^{2}-4 \alpha \beta c-\beta^{2}\right) / 32 \alpha^{2}$. Their expressions are

$$
y= \pm \frac{1}{\sqrt{-6 \alpha}}\left(\frac{6 \alpha c+\beta}{4 \alpha}-\phi\right), \quad \phi_{s} \leq \phi<\frac{6 \alpha c+\beta}{4 \alpha}
$$

Substituting (14) into $d \phi / d \xi=y$ and integrating it along the heteroclinic orbits yield equation

$$
\int_{\phi_{s}}^{\phi} \frac{d s}{((6 \alpha c+\beta) / 4 \alpha)-s}=\frac{1}{\sqrt{-6 \alpha}} \int_{0}^{\xi} d s .
$$

Completing (15) and using transformation (4), we can get a peakon solution of (3) which is the same as (13). The profiles are shown in Figures 3(c) and 3(d). 


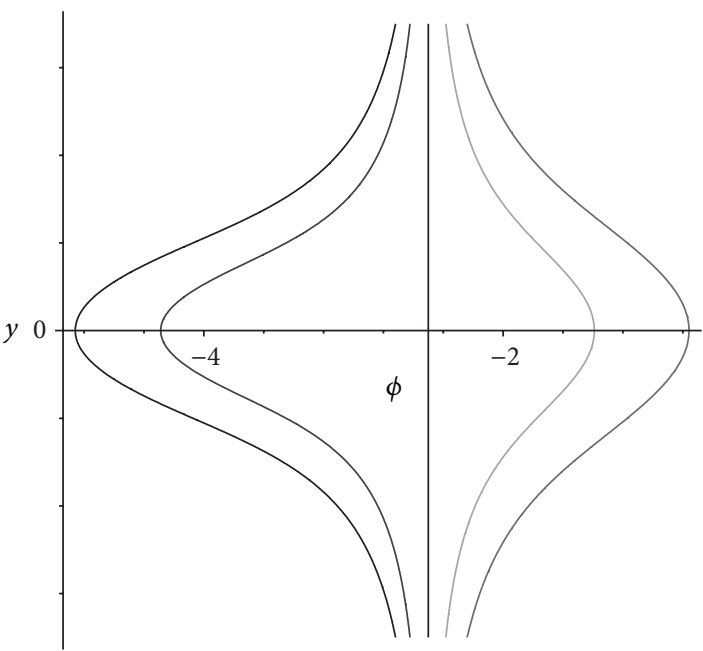

(a) $c<-\beta / 2 \alpha, g<-c^{2} / 2$

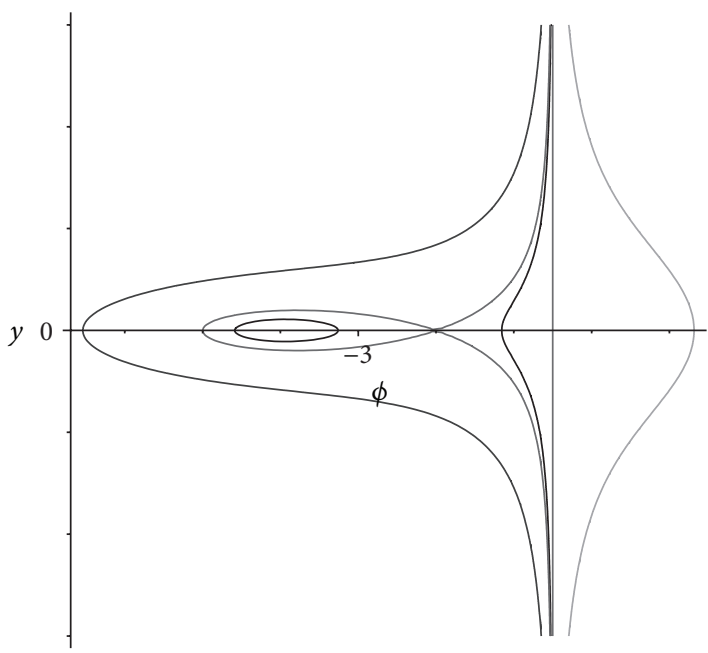

(c) $c<-\beta / 2 \alpha,-c^{2} / 2<g<\beta(4 \alpha c+\beta) / 8 \alpha^{2}$

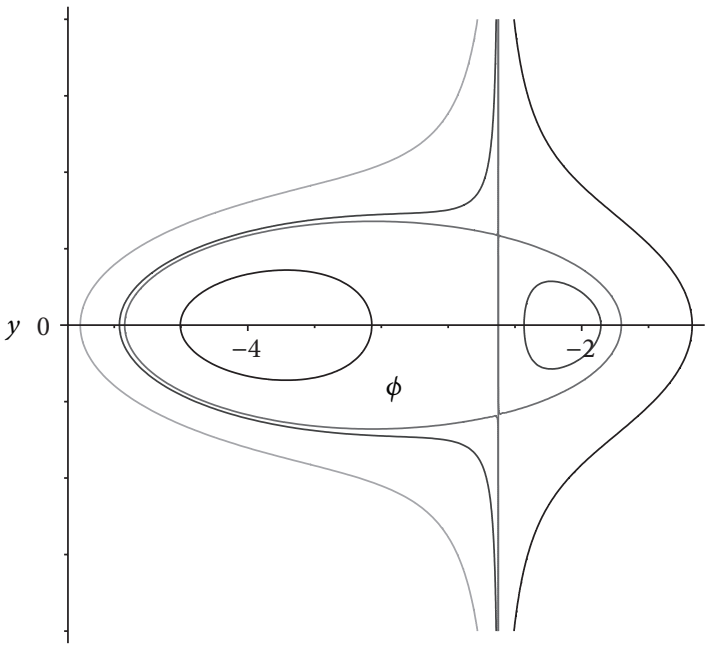

(e) $c<-\beta / 2 \alpha, g>\beta(4 \alpha c+\beta) / 8 \alpha^{2}$

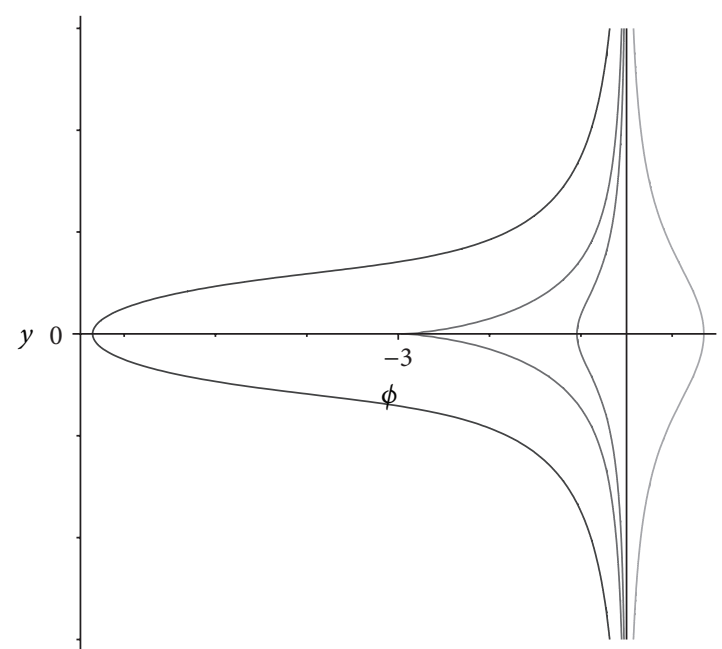

(b) $c<-\beta / 2 \alpha, g=-c^{2} / 2$

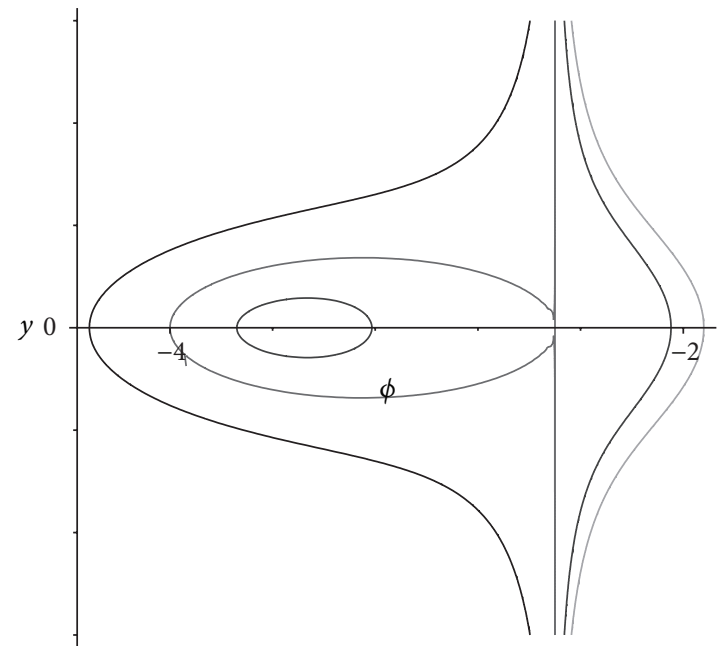

(d) $c<-\beta / 2 \alpha, g=\beta(4 \alpha c+\beta) / 8 \alpha^{2}$

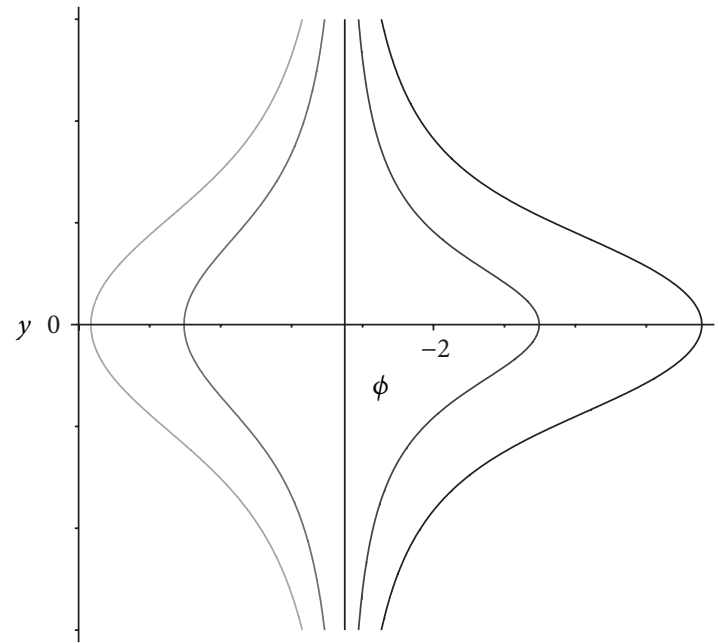

(f) $c>-\beta / 2 \alpha, g<-c^{2} / 2$

Figure 1: Continued. 


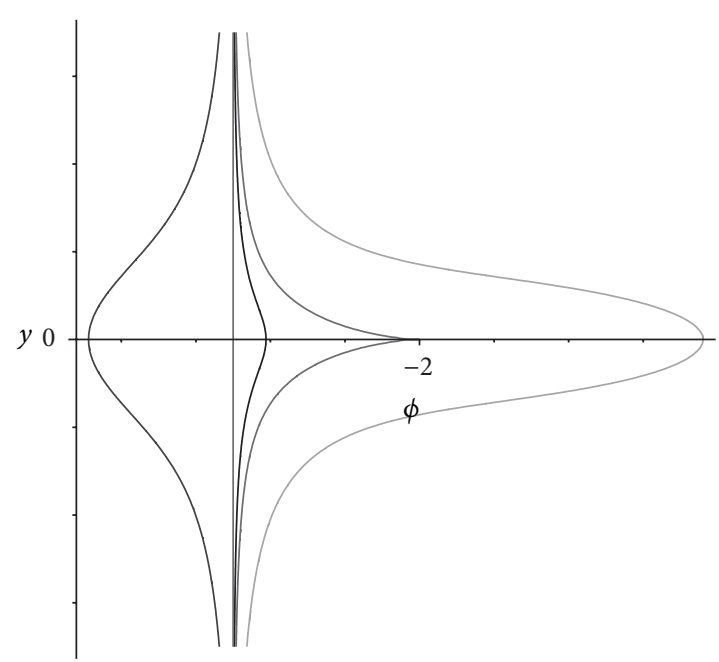

(g) $c>-\beta / 2 \alpha, g=-c^{2} / 2$

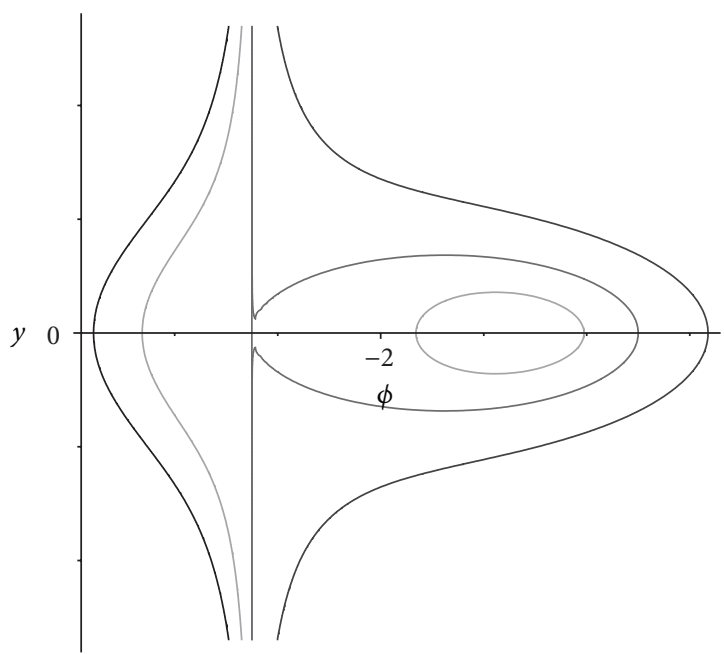

(i) $c>-\beta / 2 \alpha, g=\beta(4 \alpha c+\beta) / 8 \alpha^{2}$

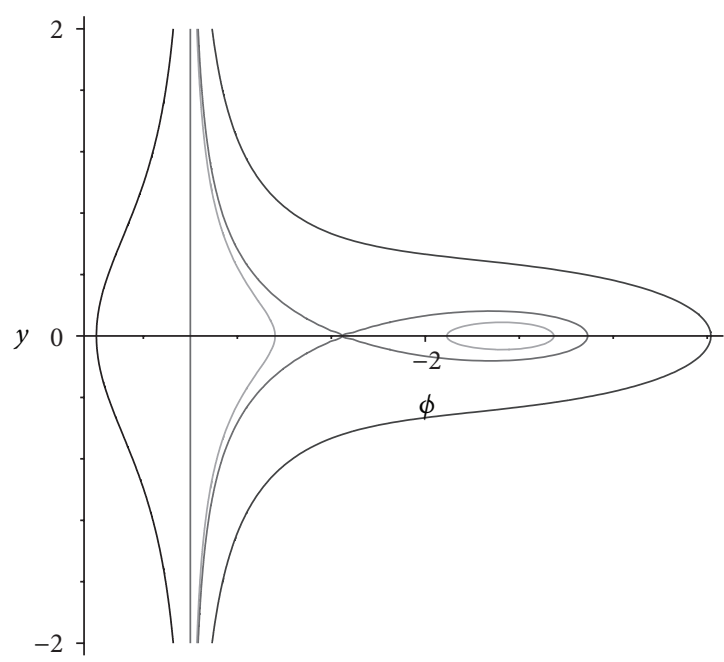

(h) $c>-\beta / 2 \alpha,-c^{2} / 2<g<\beta(4 \alpha c+\beta) / 8 \alpha^{2}$

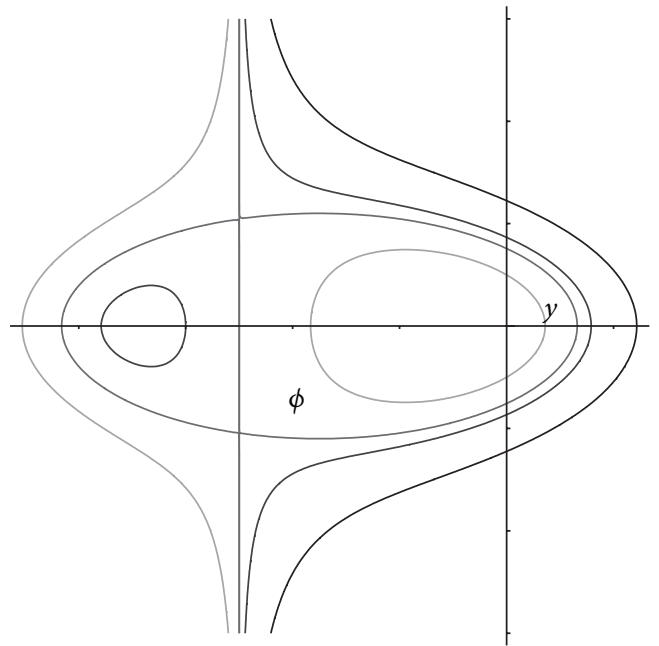

(j) $c>-\beta / 2 \alpha, g>\beta(4 \alpha c+\beta) / 8 \alpha^{2}$

FIgURE 1: Bifurcation sets and phase portraits of system (7) when $\alpha>0$.

3.2. Solitary Wave Solutions. From Figure 1(c), we see that there is one homoclinic orbit of system (7) defined by $H(\phi, y)=h_{1}$ connecting with saddle point $\left(\phi_{1}, 0\right)$ and passing point $\left(\phi_{m}, 0\right)$ when $\alpha>0, c<-\beta / 2 \alpha$, and $-c^{2} / 2<g<$ $\beta(4 \alpha c+\beta) / 8 \alpha^{2}$, where $\phi_{m}=c-2 \sqrt{2 g+c^{2}}$. Its expression is

$$
y= \pm \frac{1}{\sqrt{6 \alpha}} \frac{\phi_{1}-\phi}{\phi_{s}-\phi} \sqrt{\left(\phi-\phi_{m}\right)\left(\phi_{s}-\phi\right)}, \quad \phi_{m} \leq \phi<\phi_{1} .
$$

Substituting (16) into $d \phi / d \xi=y$ and integrating it along the homoclinic orbit yield equation

$$
\int_{\phi_{m}}^{\phi} \frac{\left(\phi_{s}-s\right) d s}{\left(\phi_{1}-s\right) \sqrt{\left(s-\phi_{m}\right)\left(\phi_{s}-s\right)}}=\frac{1}{\sqrt{6 \alpha}} \int_{0}^{\xi} d s .
$$

Completing (17) and using transformation (4), we can get a solitary wave solution of (3) as follows:

$$
\begin{gathered}
u(x, t)=\frac{\phi_{1}\left(\phi_{m} \cosh ^{2}(\omega \chi)-\phi_{s} \sinh ^{2}(\omega \chi)\right)-\phi_{m} \phi_{s}}{\left(\phi_{m} \sinh ^{2}(\omega \chi)-\phi_{s} \cosh ^{2}(\omega \chi)\right)+\phi_{1}}, \\
t=\frac{1}{c}\left(x-\sqrt{6 \alpha}\left(\chi+2 \tan ^{-1}(2 \omega \tanh (\omega \chi))\right)\right),
\end{gathered}
$$

where $\chi$ is a new parametric variable and $\omega=$ $(1 / 2) \sqrt{\left(\phi_{1}-\phi_{m}\right) /\left(\phi_{s}-\phi_{1}\right)}$. The profiles of (18) are shown in Figures 4(a) and 4(b).

From Figure 1(h), we see that there is one homoclinic orbit of system (7) defined by $H(\phi, y)=h_{2}$ connecting with saddle point $\left(\phi_{2}, 0\right)$ and passing point $\left(\phi_{M}, 0\right)$ when $\alpha>0$, 


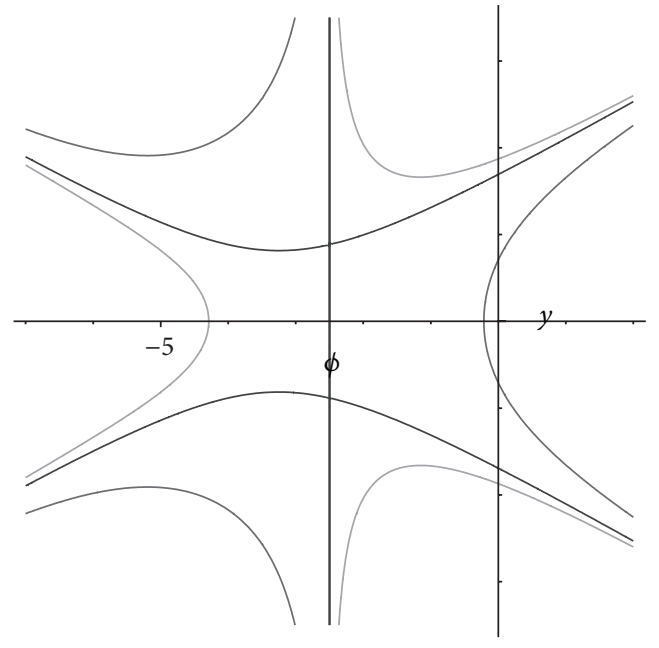

(a) $c<-\beta / 2 \alpha, g<-c^{2} / 2$

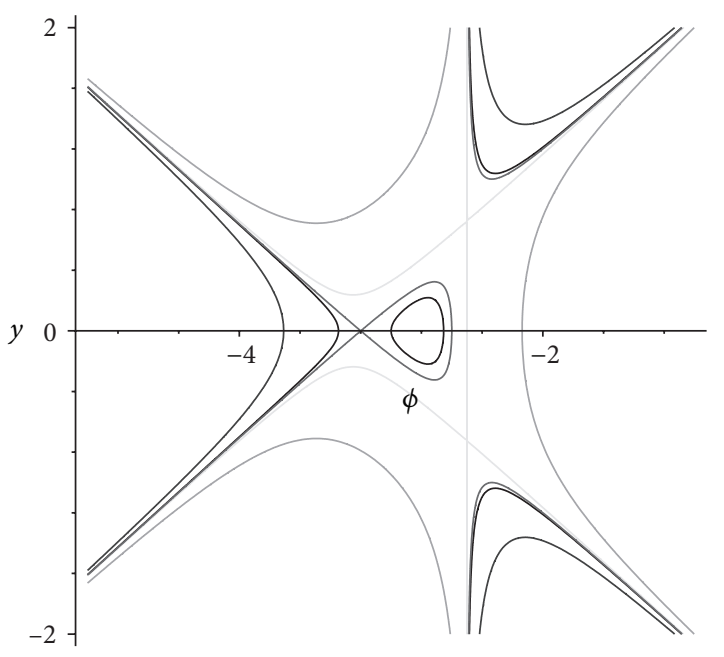

(c) $c<-\beta / 2 \alpha,-c^{2} / 2<g<-\left(12 \alpha^{2} c^{2}-4 \alpha \beta c-\beta^{2}\right) / 32 \alpha^{2}$

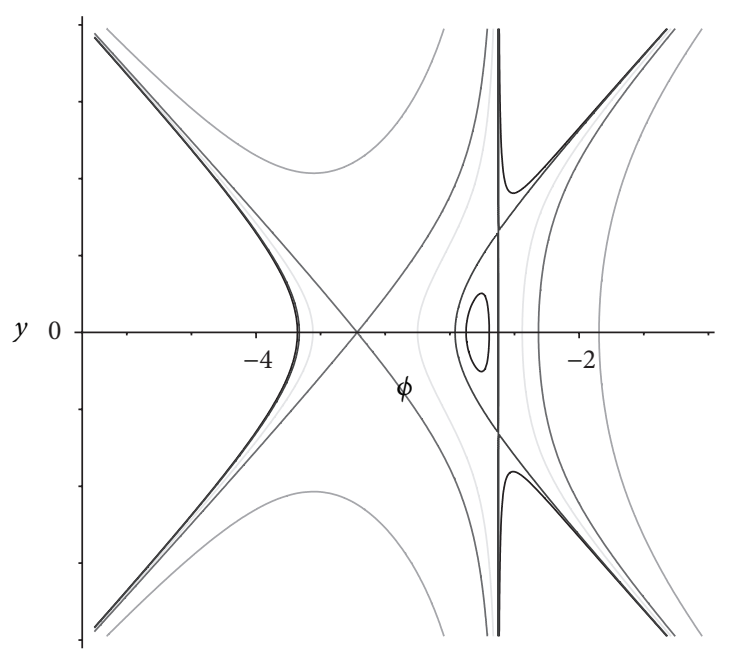

(e) $c<-\beta / 2 \alpha,-\left(12 \alpha^{2} c^{2}-4 \alpha \beta c-\beta^{2}\right) / 32 \alpha^{2}<g<\beta(4 \alpha c+$ B) $/ 8 \alpha^{2}$

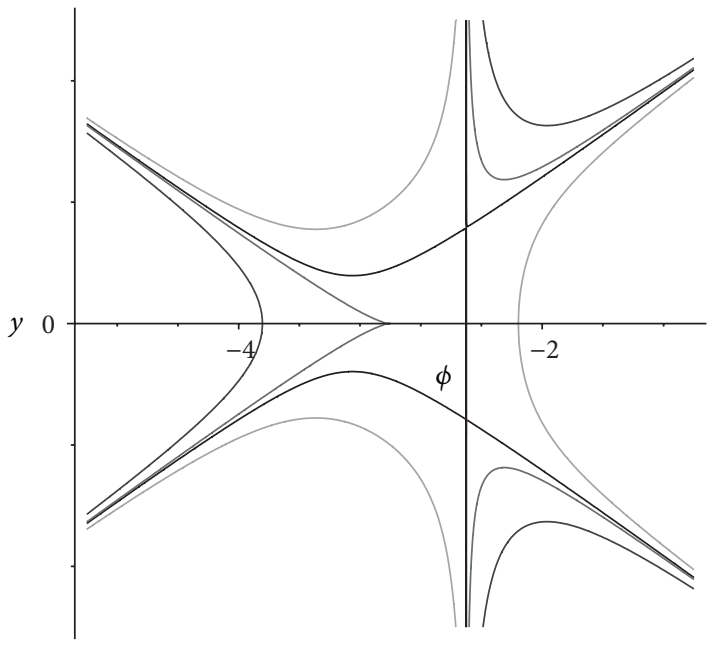

(b) $c<-\beta / 2 \alpha, g=-c^{2} / 2$

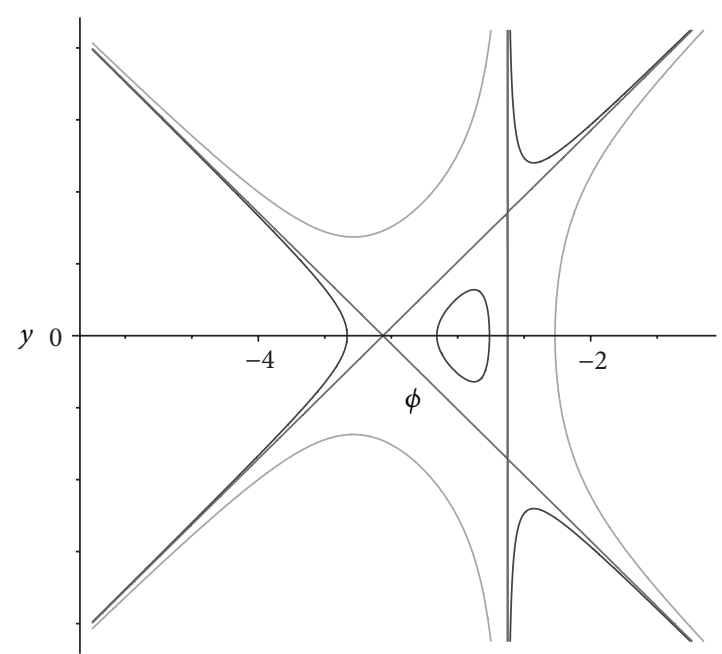

(d) $c<-\beta / 2 \alpha, g=-\left(12 \alpha^{2} c^{2}-4 \alpha \beta c-\beta^{2}\right) / 32 \alpha^{2}$

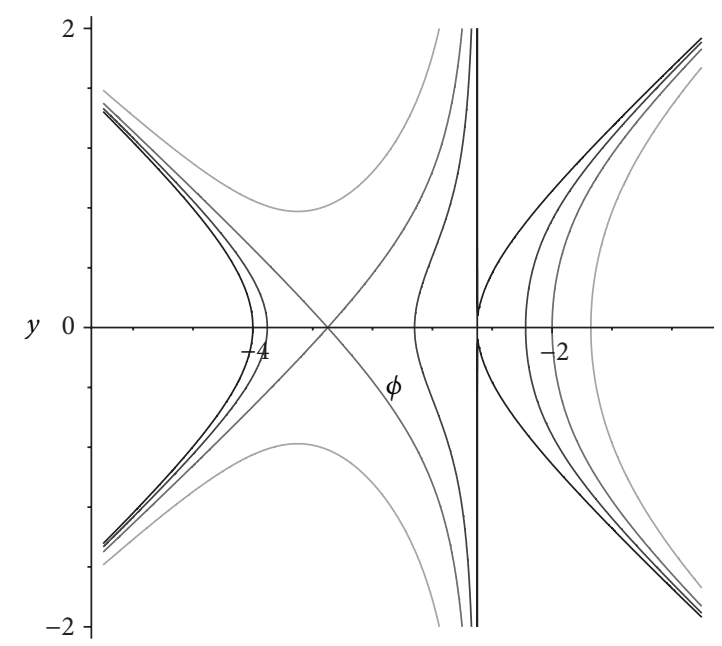

(f) $c<-\beta / 2 \alpha, g=\beta(4 \alpha c+\beta) / 8 \alpha^{2}$

Figure 2: Continued. 


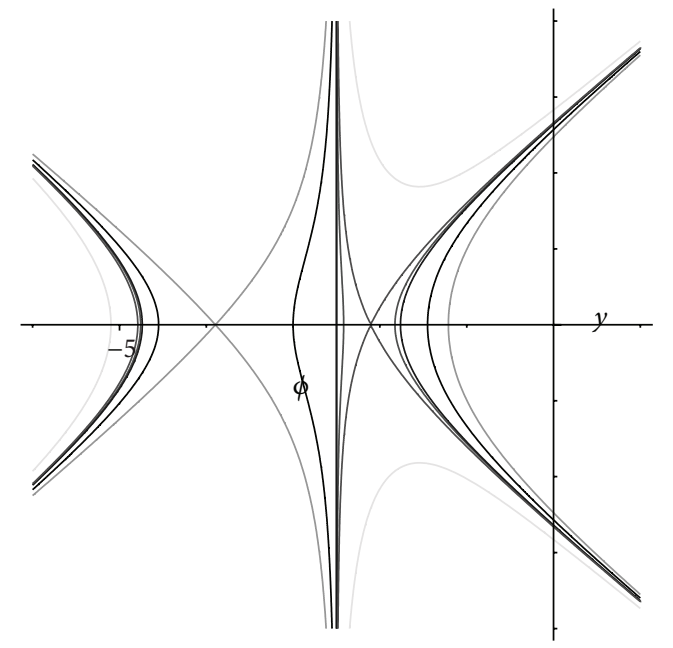

(g) $c<-\beta / 2 \alpha, g>\beta(4 \alpha c+\beta) / 8 \alpha^{2}$

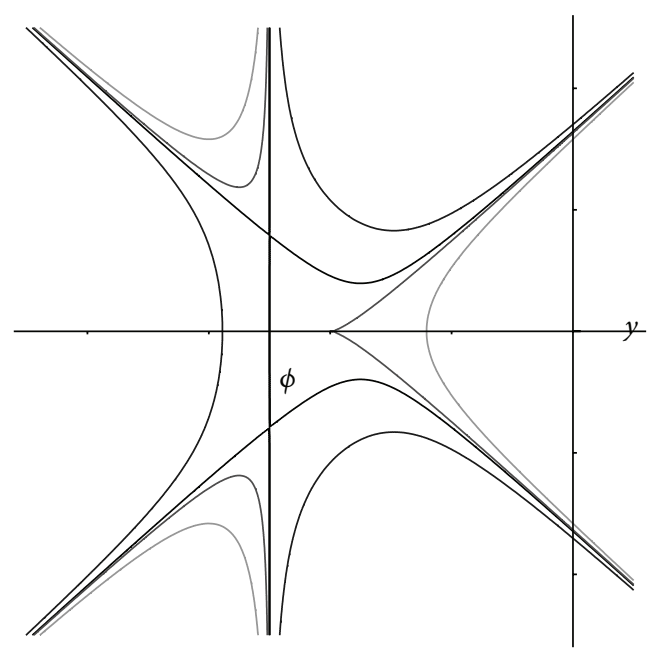

(i) $c>-\beta / 2 \alpha, g=-c^{2} / 2$

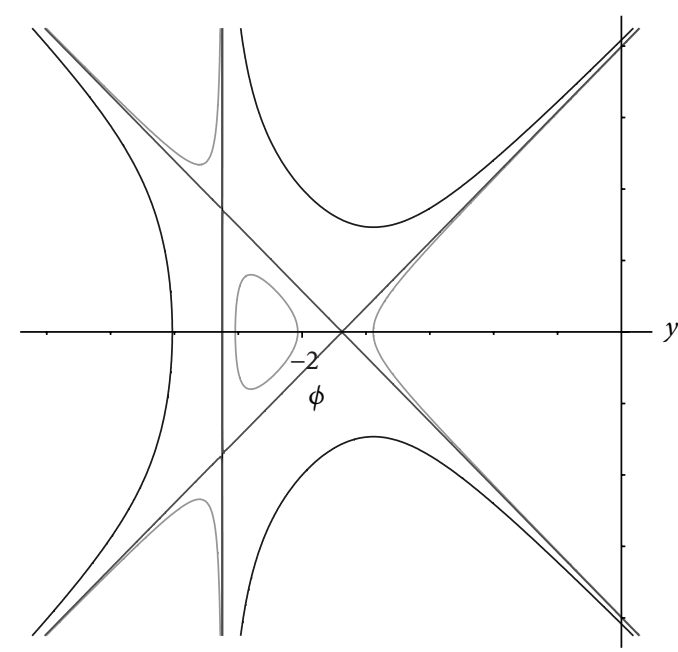

(k) $c>-\beta / 2 \alpha, g=-\left(12 \alpha^{2} c^{2}-4 \alpha \beta c-\beta^{2}\right) / 32 \alpha^{2}$

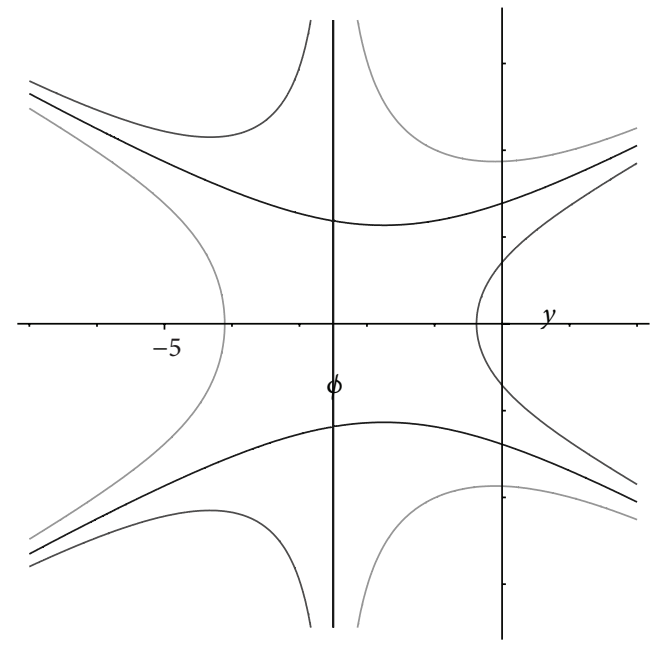

(h) $c>-\beta / 2 \alpha, g<-c^{2} / 2$

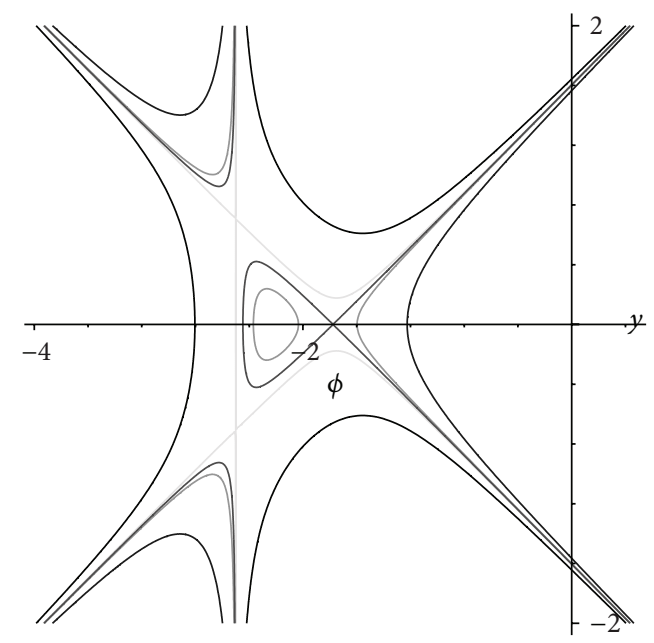

(j) $c>-\beta / 2 \alpha,-c^{2} / 2<g<-\left(12 \alpha^{2} c^{2}-4 \alpha \beta c-\beta^{2}\right) / 32 \alpha^{2}$

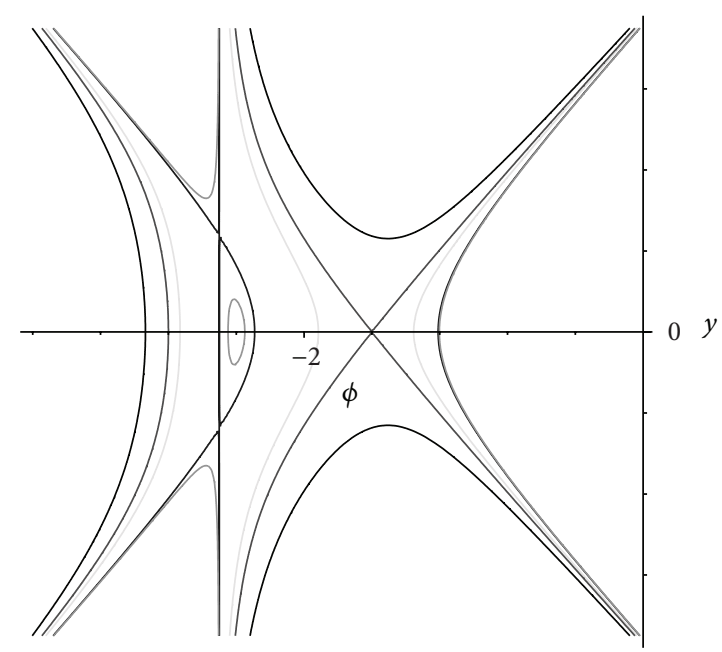

(l) $c>-\beta / 2 \alpha,-\left(12 \alpha^{2} c^{2}-4 \alpha \beta c-\beta^{2}\right) / 32 \alpha^{2}<g<\beta(4 \alpha c+$ $\beta) / 8 \alpha^{2}$

FIgUre 2: Continued. 


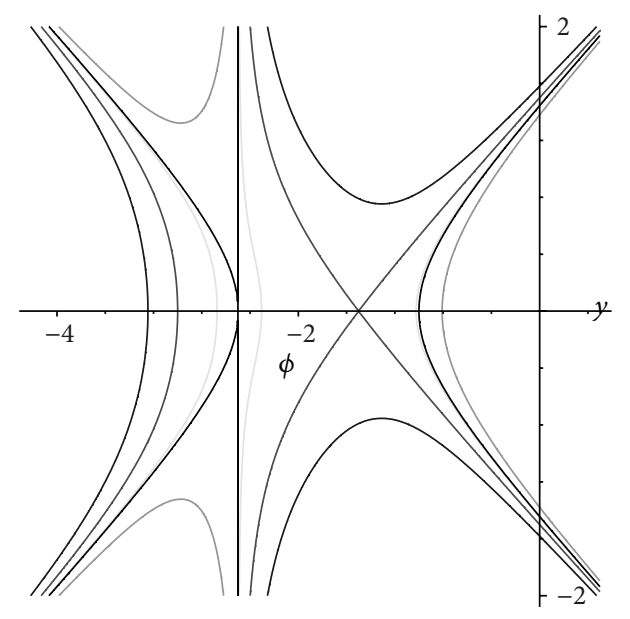

(m) $c>-\beta / 2 \alpha, g=\beta(4 \alpha c+\beta) / 8 \alpha^{2}$

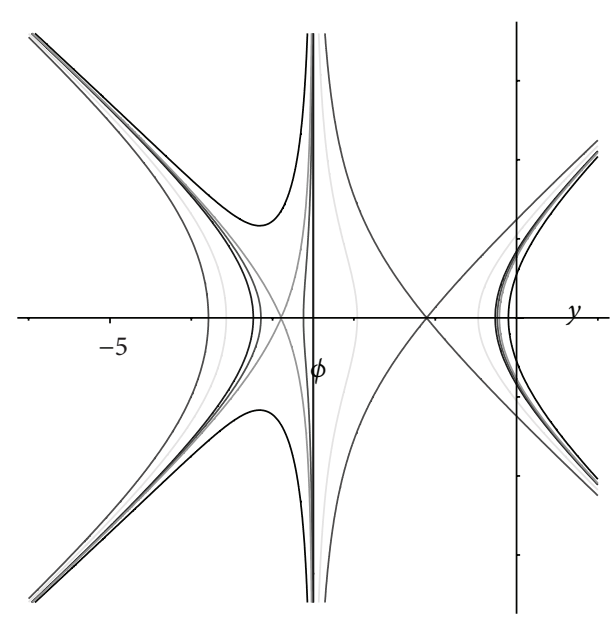

(n) $c>-\beta / 2 \alpha, g>\beta(4 \alpha c+\beta) / 8 \alpha^{2}$

FIGURE 2: Bifurcation sets and phase portraits of system (7) when $\alpha<0$.

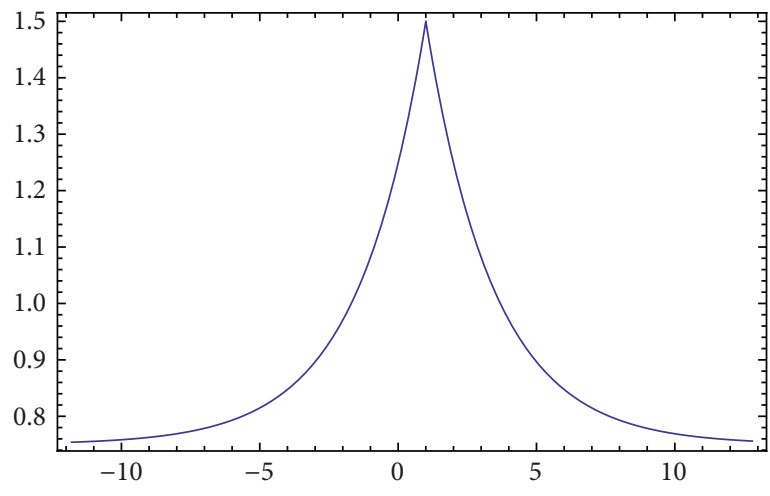

(a) $\alpha=-1, \beta=3, c=1$

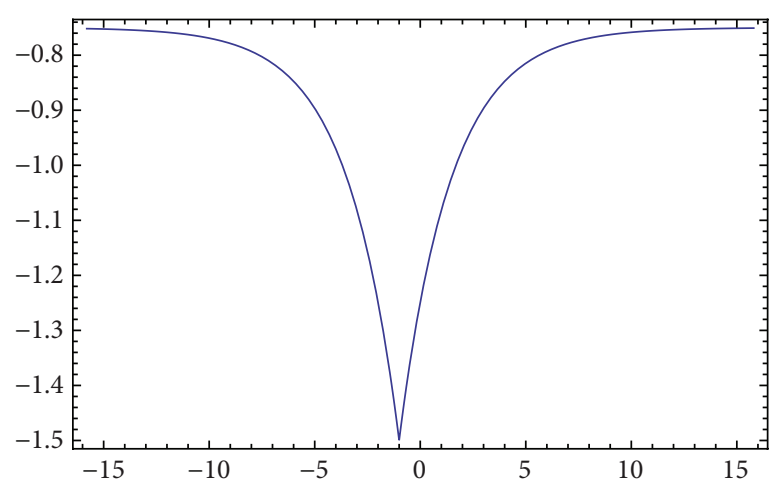

(c) $\alpha=-1, \beta=-3, c=-1$

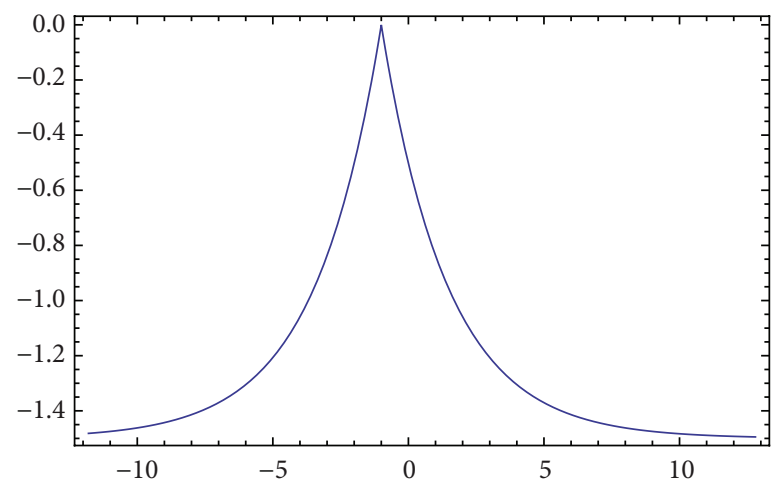

(b) $\alpha=-1, \beta=0, c=-1$

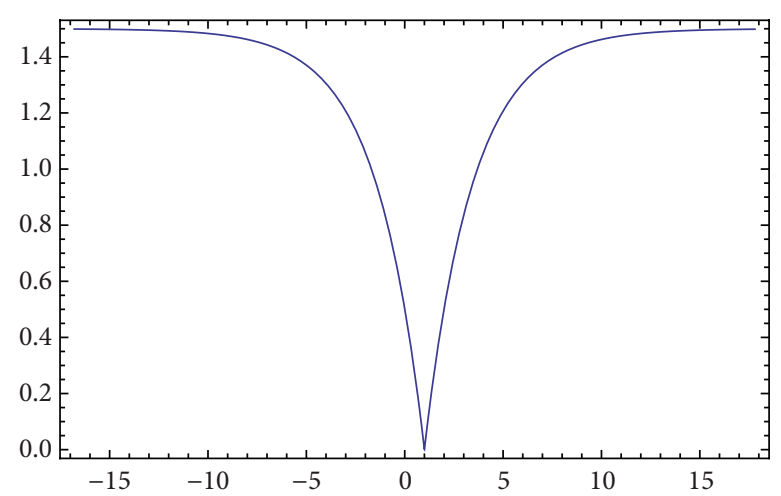

(d) $\alpha=-1, \beta=0, c=1$

Figure 3: Peakons of (3) for $x=1$.

$c>-\beta / 2 \alpha$, and $-c^{2} / 2<g<\beta(4 \alpha c+\beta) / 8 \alpha^{2}$, where $\phi_{M}=c+2 \sqrt{2 g+c^{2}}$. Its expression is

$$
y= \pm \frac{1}{\sqrt{6 \alpha}} \frac{\phi-\phi_{2}}{\phi-\phi_{s}} \sqrt{\left(\phi_{M}-\phi\right)\left(\phi-\phi_{s}\right)}, \quad \phi_{2}<\phi \leq \phi_{M} .
$$

Substituting (19) into $d \phi / d \xi=y$ and integrating it along the homoclinic orbit yield equation

$$
\int_{\phi}^{\phi_{M}} \frac{\left(s-\phi_{s}\right) d s}{\left(s-\phi_{2}\right) \sqrt{\left(\phi_{M}-s\right)\left(s-\phi_{s}\right)}}=\frac{1}{\sqrt{6 \alpha}} \int_{0}^{\xi} d s .
$$




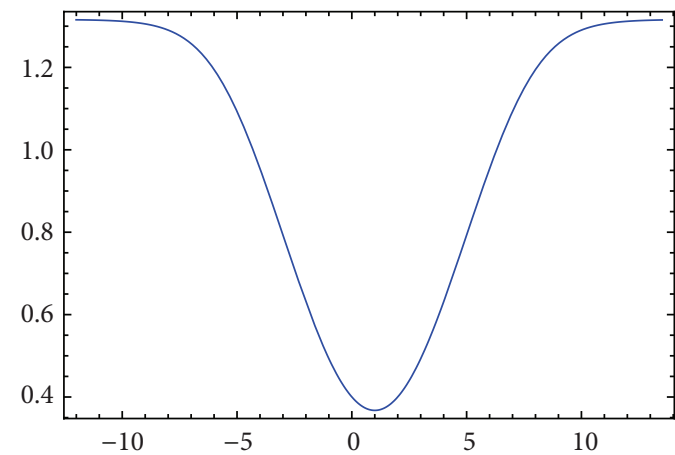

(a) $\alpha=1, \beta=-3, c=1, g=-0.45$

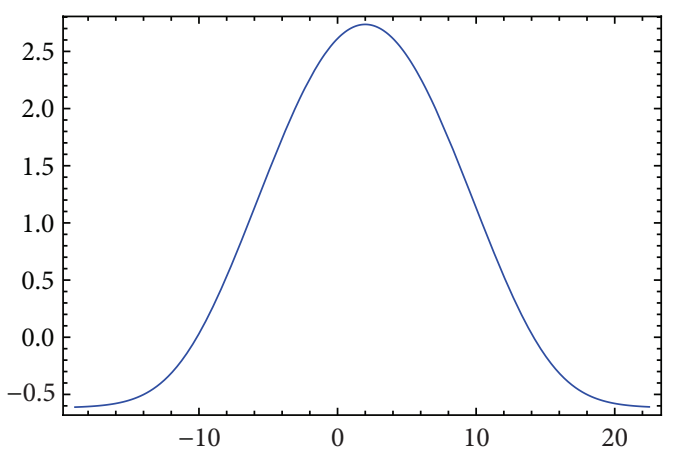

(c) $\alpha=1, \beta=3, c=-1, g=-0.4$

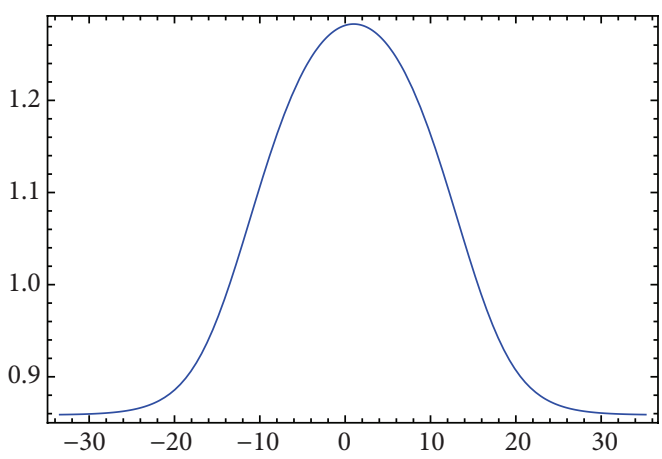

(e) $\alpha=-1, \beta=3, c=1, g=-0.49$

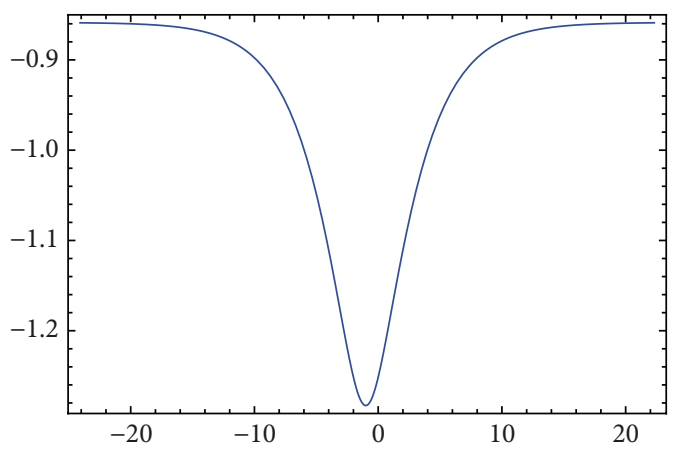

(g) $\alpha=-1, \beta=-3, c=-1, g=-0.49$

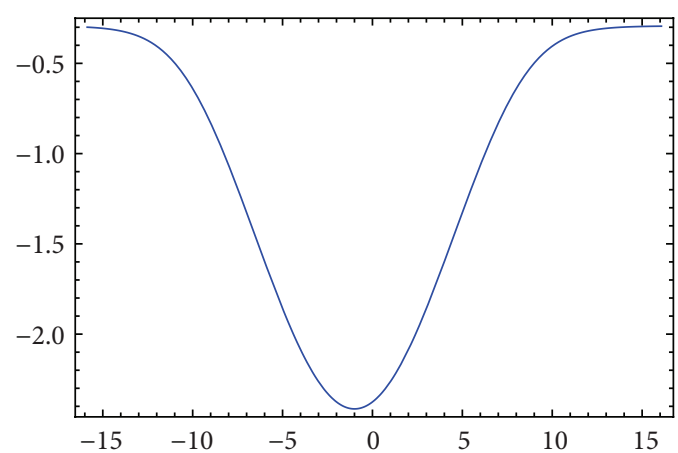

(b) $\alpha=1, \beta=0, c=-2, g=-1$

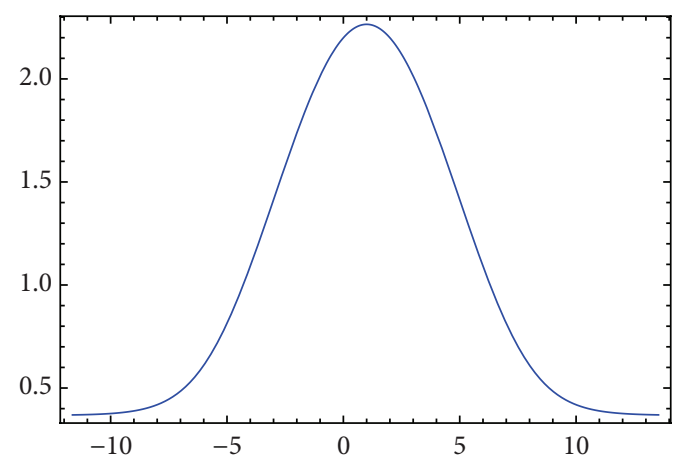

(d) $\alpha=1, \beta=0, c=2, g=-1$

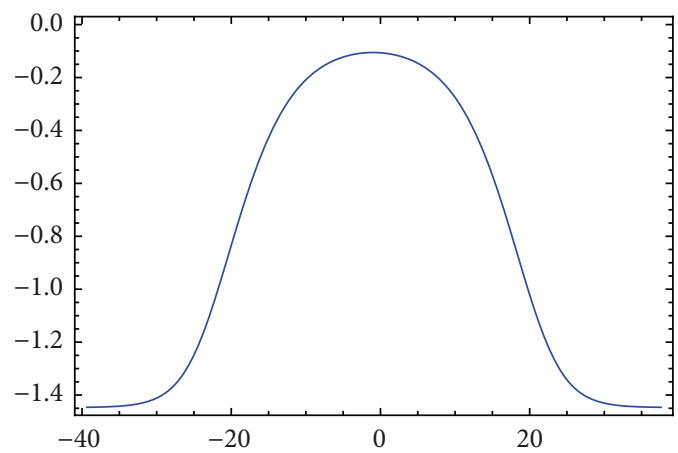

(f) $\alpha=-1, \beta=0, c=-1, g=-0.4$

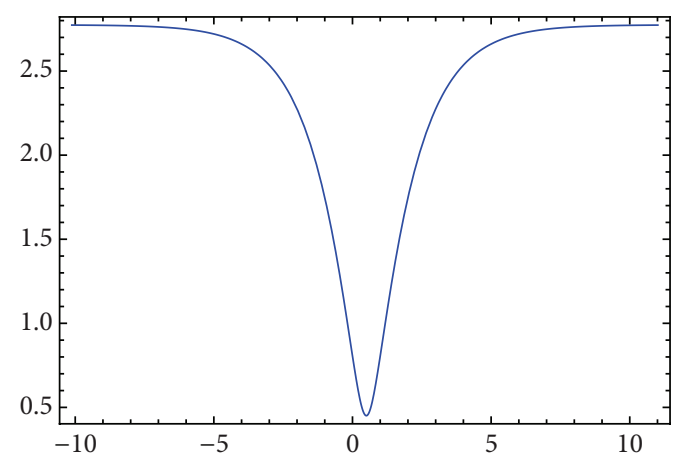

(h) $\alpha=-1, \beta=0, c=2, g=-1.7$

FIGURE 4: Solitary waves of (3) for $x=1$. 
Completing (20) and using transformation (4), we can get a solitary wave solution of (3) as follows:

$$
\begin{gathered}
u(x, t)=\frac{\phi_{2}\left(\phi_{M} \cosh ^{2}(\omega \chi)-\phi_{s} \sinh ^{2}(\omega \chi)\right)-\phi_{M} \phi_{s}}{\left(\phi_{M} \sinh ^{2}(\omega \chi)-\phi_{s} \cosh ^{2}(\omega \chi)\right)+\phi_{2}}, \\
t=\frac{1}{c}\left(x-\sqrt{6 \alpha}\left(\chi-2 \tan ^{-1}(2 \omega \tanh (\omega \chi))\right)\right),
\end{gathered}
$$

where $\omega=(1 / 2) \sqrt{\left(\phi_{M}-\phi_{2}\right) /\left(\phi_{2}-\phi_{s}\right)}$. The profiles of (21) are shown in Figures $4(\mathrm{c})$ and $4(\mathrm{~d})$.

From Figure 2(c), we see that there is one homoclinic orbit of system (7) defined by $H(\phi, y)=h_{2}$ connecting with saddle point $\left(\phi_{2}, 0\right)$ and passing point $\left(\phi_{M}, 0\right)$ when $\alpha<0$, $c<-\beta / 2 \alpha$, and $-c^{2} / 2<g<-\left(12 \alpha^{2} c^{2}-4 \alpha \beta c-\beta^{2}\right) / 32 \alpha^{2}$, where $\phi_{M}=c+2 \sqrt{2 g+c^{2}}$. Its expression is

$$
y= \pm \frac{1}{\sqrt{-6 \alpha}} \frac{\phi-\phi_{2}}{\phi_{s}-\phi} \sqrt{\left(\phi_{M}-\phi\right)\left(\phi_{s}-\phi\right)}, \quad \phi_{2}<\phi \leq \phi_{M} .
$$

Substituting (22) into $d \phi / d \xi=y$ and integrating it along the homoclinic orbit yield equation

$$
\int_{\phi}^{\phi_{M}} \frac{\left(\phi_{s}-s\right) d s}{\left(s-\phi_{2}\right) \sqrt{\left(\phi_{M}-s\right)\left(\phi_{s}-s\right)}}=\frac{1}{\sqrt{-6 \alpha}} \int_{0}^{\xi} d s
$$

Completing (23) and using transformation (4), we can get a solitary wave solution of (3) as follows:

$$
\begin{gathered}
u(x, t)=\frac{\phi_{2}\left(\phi_{M} \cosh ^{2}(\omega \chi)-\phi_{s} \sinh ^{2}(\omega \chi)\right)-\phi_{M} \phi_{s}}{\left(\phi_{M} \sinh ^{2}(\omega \chi)-\phi_{s} \cosh ^{2}(\omega \chi)\right)+\phi_{2}}, \\
t=\frac{1}{c}\left(x-\sqrt{-6 \alpha}\left(\chi+2 \tanh ^{-1}(2 \omega \tanh (\omega \chi))\right)\right),
\end{gathered}
$$

where $\omega=(1 / 2) \sqrt{\left(\phi_{M}-\phi_{2}\right) /\left(\phi_{s}-\phi_{2}\right)}$. The profiles of (24) are shown in Figures 4(e) and 4(f).

From Figure 2(j), we see that there is one homoclinic orbit of system (7) defined by $H(\phi, y)=h_{1}$ connecting with saddle point $\left(\phi_{1}, 0\right)$ and passing point $\left(\phi_{m}, 0\right)$ when $\alpha<0$, $c>-\beta / 2 \alpha,-c^{2} / 2<g<-\left(12 \alpha^{2} c^{2}-4 \alpha \beta c-\beta^{2}\right) / 32 \alpha^{2}$, where $\phi_{m}=c-2 \sqrt{2 g+c^{2}}$. Its expression is

$$
y= \pm \frac{1}{\sqrt{-6 \alpha}} \frac{\phi_{1}-\phi}{\phi-\phi_{s}} \sqrt{\left(\phi-\phi_{m}\right)\left(\phi-\phi_{s}\right)}, \quad \phi_{m} \leq \phi<\phi_{1} .
$$

Substituting (25) into $d \phi / d \xi=y$ and integrating it along the homoclinic orbit yield equation

$$
\int_{\phi_{m}}^{\phi} \frac{\left(s-\phi_{s}\right) d s}{\left(\phi_{1}-s\right) \sqrt{\left(s-\phi_{m}\right)\left(s-\phi_{s}\right)}}=\frac{1}{\sqrt{-6 \alpha}} \int_{0}^{\xi} d s
$$

Completing (26) and using transformation (4), we can get a solitary wave solution of (3) as follows:

$$
\begin{aligned}
& u(x, t)=\frac{\phi_{1}\left(\phi_{m} \cosh ^{2}(\omega \chi)-\phi_{s} \sinh ^{2}(\omega \chi)\right)-\phi_{m} \phi_{s}}{\left(\phi_{m} \sinh ^{2}(\omega \chi)-\phi_{s} \cosh ^{2}(\omega \chi)\right)+\phi_{1}} \\
& t=\frac{1}{c}\left(x-\sqrt{-6 \alpha}\left(\chi-2 \tanh ^{-1}(2 \omega \tanh (\omega \chi))\right)\right),
\end{aligned}
$$

where $\omega=(1 / 2) \sqrt{\left(\phi_{1}-\phi_{m}\right) /\left(\phi_{1}-\phi_{s}\right)}$. The profiles of (27) are shown in Figures 4(g) and 4(h).

3.3. Smooth Periodic Wave Solutions. From Figure 1(d), we see that there is a periodic orbit of system (7) defined by $H(\phi, y)=\beta^{2}(3 \alpha c+\beta) / 12 \alpha^{3}$ enclosing the center point $((4 \alpha c+\beta) / 2 \alpha, 0)$ and passing points $\left(\phi_{s}, 0\right)$ and $((3 \alpha c+\beta) / \alpha, 0)$ when $\alpha>0, c<-\beta / 2 \alpha$, and $g=\beta(4 \alpha c+\beta) / 8 \alpha^{2}$. Its expression is

$$
y= \pm \frac{1}{\sqrt{6 \alpha}} \sqrt{\left(\phi-\frac{3 \alpha c+\beta}{\alpha}\right)\left(\phi_{s}-\phi\right)}, \quad \frac{3 \alpha c+\beta}{\alpha} \leq \phi \leq \phi_{s} .
$$

Substituting (28) into $d \phi / d \xi=y$ and integrating it along the periodic orbit yield equation

$$
\int_{(3 \alpha c+\beta) / \alpha}^{\phi} \frac{d s}{\sqrt{(s-((3 \alpha c+\beta) / \alpha))\left(\phi_{s}-s\right)}}=\frac{1}{\sqrt{6 \alpha}} \int_{0}^{\xi} d s .
$$

Completing (29) and using transformation (4), we can get a smooth periodic wave solution of (3) as follows:

$$
u(x, t)=\phi_{s} \sin ^{2}(\omega(x-c t))+\frac{3 \alpha c+\beta}{\alpha} \cos ^{2}(\omega(x-c t)),
$$

where $\omega=1 / 2 \sqrt{6 \alpha}$. The profiles of (30) are shown in Figures $5(\mathrm{a})$ and 5(b).

From Figure 1(i), we see that there is a periodic orbit of system (7) defined by $H(\phi, y)=\beta^{2}(3 \alpha c+\beta) / 12 \alpha^{3}$ enclosing the center point $((4 \alpha c+\beta) / 2 \alpha, 0)$ and passing points $\left(\phi_{s}, 0\right)$ and $((3 \alpha c+\beta) / \alpha, 0)$ when $\alpha>0, c>-\beta / 2 \alpha$, and $g=\beta(4 \alpha c+$ $\beta) / 8 \alpha^{2}$. Its expression is

$$
y= \pm \frac{1}{\sqrt{6 \alpha}} \sqrt{\left(\frac{3 \alpha c+\beta}{\alpha}-\phi\right)\left(\phi-\phi_{s}\right)}, \quad \phi_{s} \leq \phi \leq \frac{3 \alpha c+\beta}{\alpha} .
$$

Substituting (31) into $d \phi / d \xi=y$ and integrating it along the periodic orbit yield equation

$$
\int_{\phi}^{(3 \alpha c+\beta) / \alpha} \frac{d s}{\sqrt{(((3 \alpha c+\beta) / \alpha)-s)\left(s-\phi_{s}\right)}}=\frac{1}{\sqrt{6 \alpha}} \int_{0}^{\xi} d s .
$$




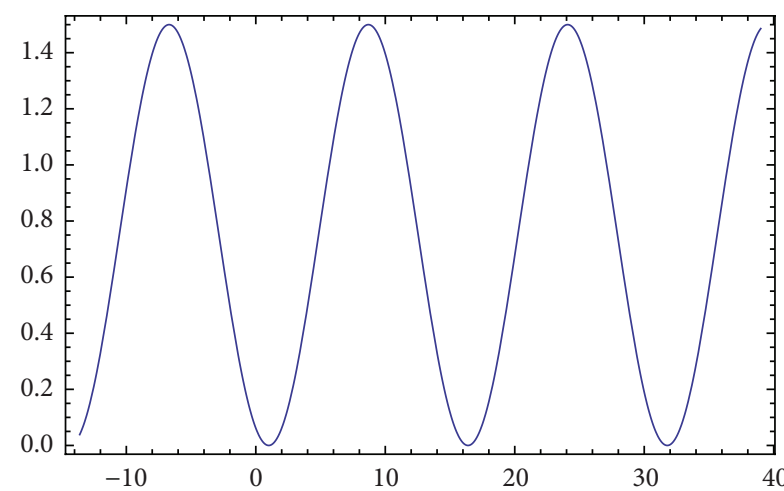

(a) $\alpha=1, \beta=-3, c=1$

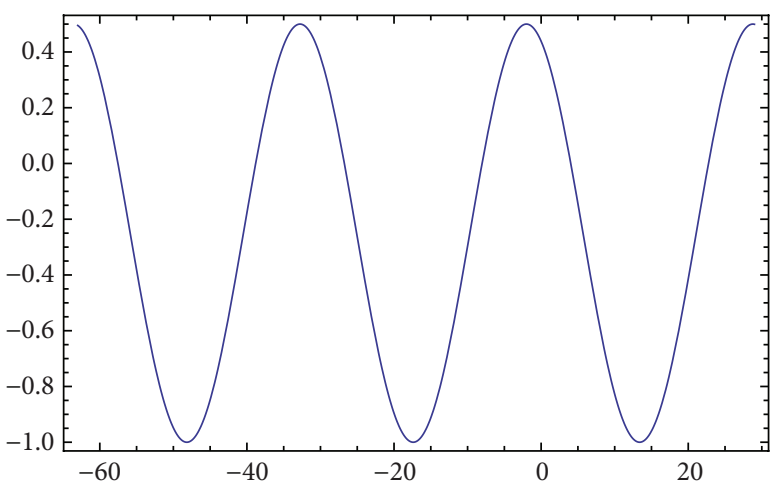

(c) $\alpha=1, \beta=2, c=-0.5$

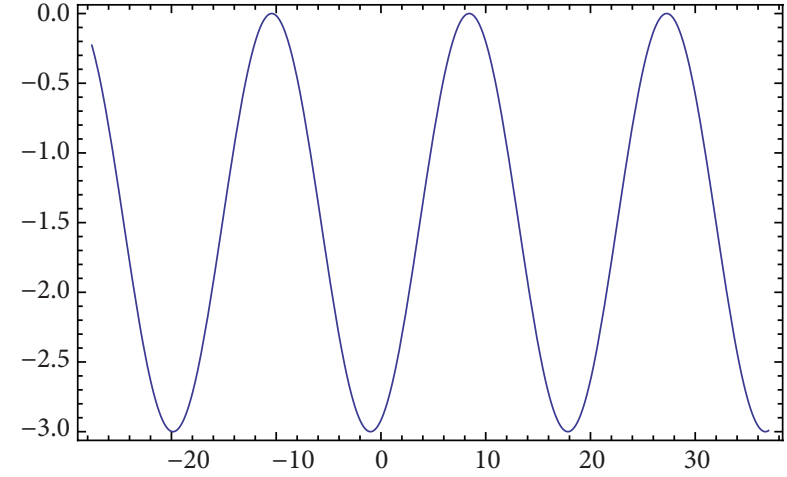

(b) $\alpha=1.5, \beta=0, c=-1$

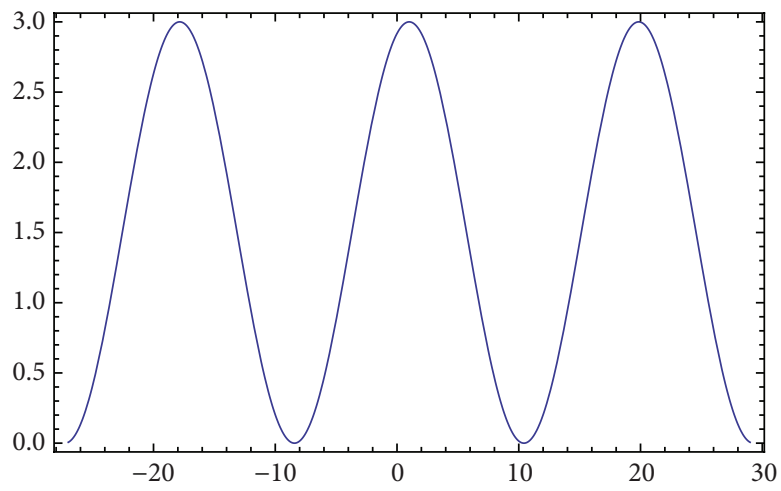

(d) $\alpha=1.5, \beta=0, c=1$

Figure 5: Smooth periodic waves of (3) for $x=1$.

Completing (32) and using transformation (4), we can get a smooth periodic wave solution of (3) which is the same as (30). The profiles are shown in Figures 5(c) and 5(d).

3.4. Compacton Solutions. For given $h \in\left(h_{1}, h_{s}\right), h_{s}<h_{2}$ (or $\left.h \in\left(h_{1}, h_{2}\right), h_{s}>h_{2}\right)$ in Figure 1(c), the level curve defined by $H(\phi, y)=h$ is shown in Figure 6(a).

For given $h \in\left(h_{s}, h_{2}\right), h_{1}<h_{s}$ in Figure 1(c), $h \in\left(h_{1}, h_{2}\right)$ in Figure 1(d), and $h \in\left(h_{s}, h_{2}\right)$ in Figures 1(e) and 1(j), respectively, the level curve defined by $H(\phi, y)=h$ is shown in Figure 6(b).

For given $h \in\left(h_{s}, h_{2}\right), h_{s}>h_{1}\left(\right.$ or $\left.h \in\left(h_{1}, h_{2}\right), h_{s}<h_{1}\right)$ in Figure 1(h), the level curve defined by $H(\phi, y)=h$ is shown in Figure 6(c).

For given $h \in\left(h_{1}, h_{s}\right), h_{s}<h_{2}$ in Figure 1(h), and $h \in$ $\left(h_{1}, h_{2}\right)$ in Figure 1(i), $h \in\left(h_{1}, h_{s}\right)$ in Figures 1(e) and 1(j), respectively, the level curve defined by $H(\phi, y)=h$ is shown in Figure 6(d).

For given $h \in\left(h_{s}, h_{2}\right)$ in Figures 2(e), 2(f), 2(g), and 2(n), respectively, the level curve defined by $H(\phi, y)=h$ is shown in Figure 6(e).

For given $h \in\left(h_{1}, h_{s}\right)$ in Figures 2(g), 2(l), 2(m), and 2(n), respectively, the level curve defined by $H(\phi, y)=h$ is shown in Figure 6(f).
From Figure 6(a), we see that there are a periodic orbit and an open curve of system (7) defined by $H(\phi, y)=h$ when $\alpha>0, c<-\beta / 2 \alpha,-c^{2} / 2<g<\beta(4 \alpha c+\beta) / 8 \alpha^{2}, h \in\left(h_{1}, h_{s}\right)$, $h_{s}<h_{2}\left(\right.$ or $h \in\left(h_{1}, h_{2}\right)$, and $\left.h_{s}>h_{2}\right)$. The open curve passes point $\left(\gamma_{1}, 0\right)$ and approaches the line $\phi=\phi_{s}$; its expression is

$$
y= \pm \frac{1}{\sqrt{6 \alpha}} \sqrt{\frac{\left(\phi-\gamma_{1}\right)\left(\phi-\gamma_{2}\right)\left(\phi-\gamma_{3}\right)}{\phi_{s}-\phi}}, \quad \gamma_{1} \leq \phi<\phi_{s}
$$

where $\gamma_{1}, \gamma_{2}$, and $\gamma_{3}\left(\gamma_{3}<\gamma_{2}<\gamma_{1}<\phi_{s}\right)$ are three real roots of $\psi^{3}-3 c \psi^{2}-6 g \psi-3 h=0$. For example, $\gamma_{1} \approx$ $-2.681985997, \gamma_{2} \approx-2.948887226, \gamma_{3} \approx-3.369126778$ when $\alpha=0.2, \beta=1, c=-3, g=-4.48$, and $h=-8.882$, and $\gamma_{1} \approx-0.06941350133, \gamma_{2} \approx-0.5430641149$, and $\gamma_{3} \approx$ -2.387522384 when $\alpha=1, \beta=0, c=-1, g=-0.25$, and $h=-0.03$.

Substituting (33) into $d \phi / d \xi=y$ and integrating it along the open curve yield equation

$$
\int_{\gamma_{1}}^{\phi} \sqrt{\frac{\phi_{s}-s}{\left(s-\gamma_{1}\right)\left(s-\gamma_{2}\right)\left(s-\gamma_{3}\right)}} d s=\frac{1}{\sqrt{6 \alpha}} \int_{0}^{\xi} d s .
$$


Mathematical Problems in Engineering

11

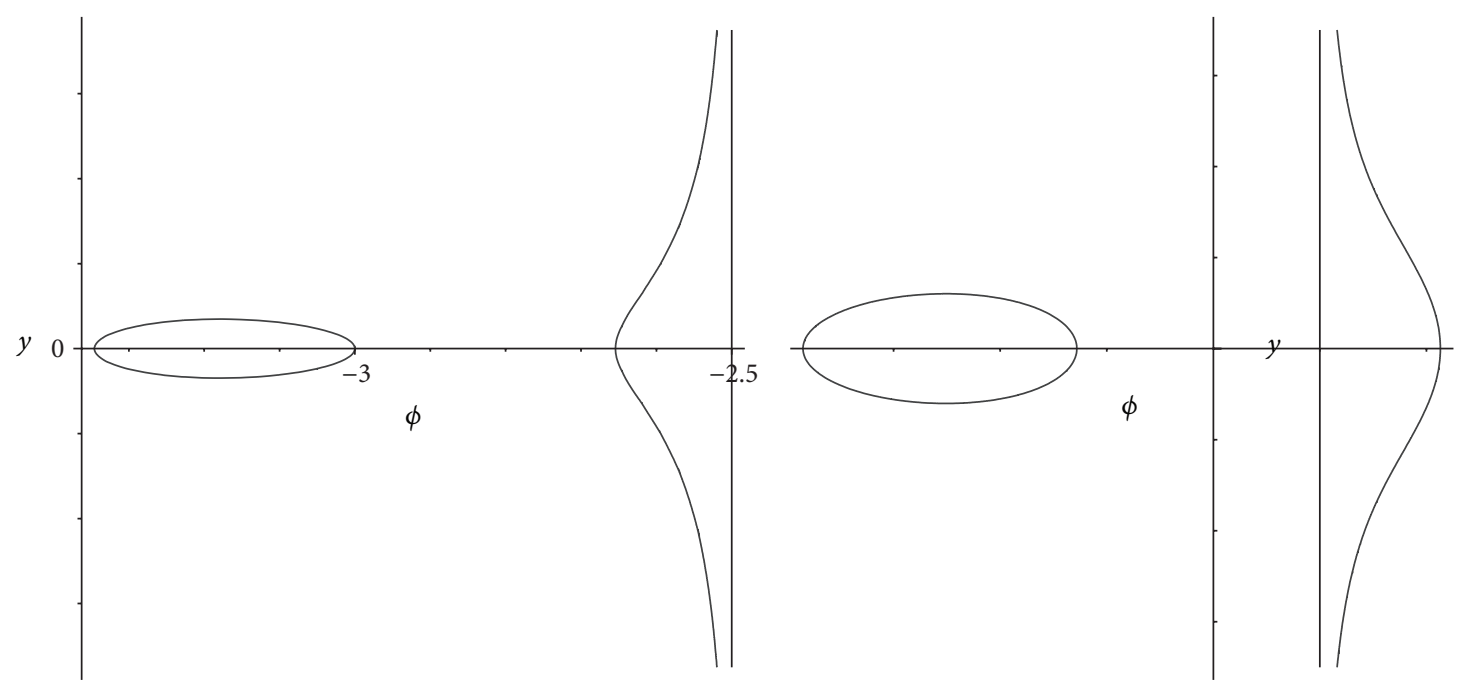

(a)

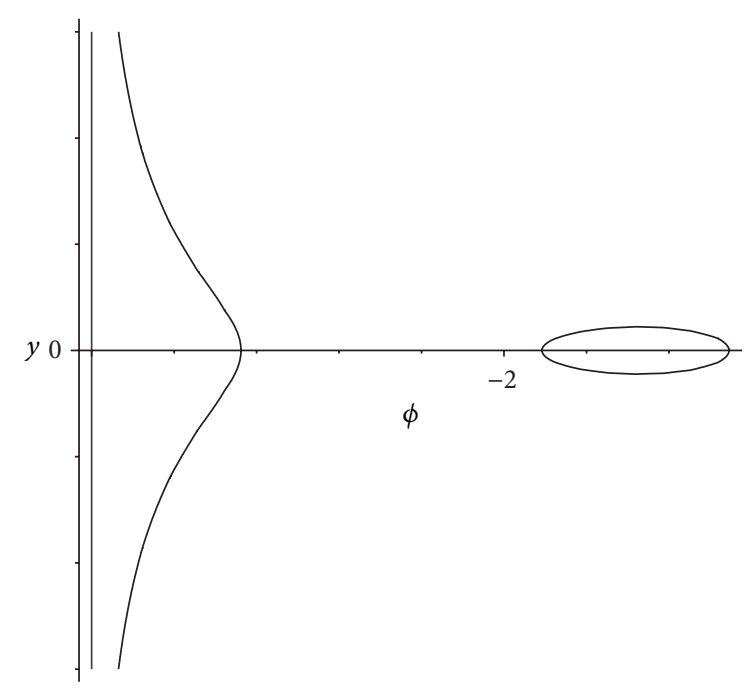

(c)

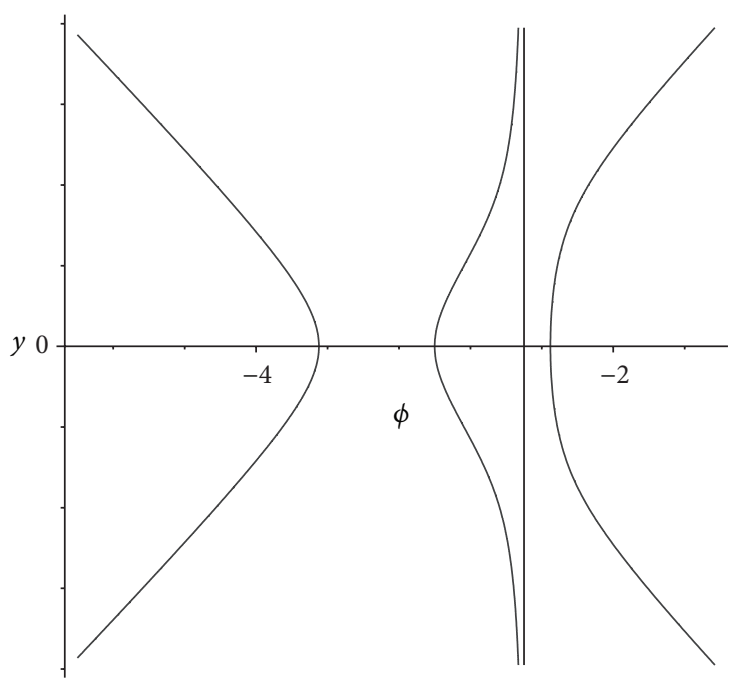

(e) (b)

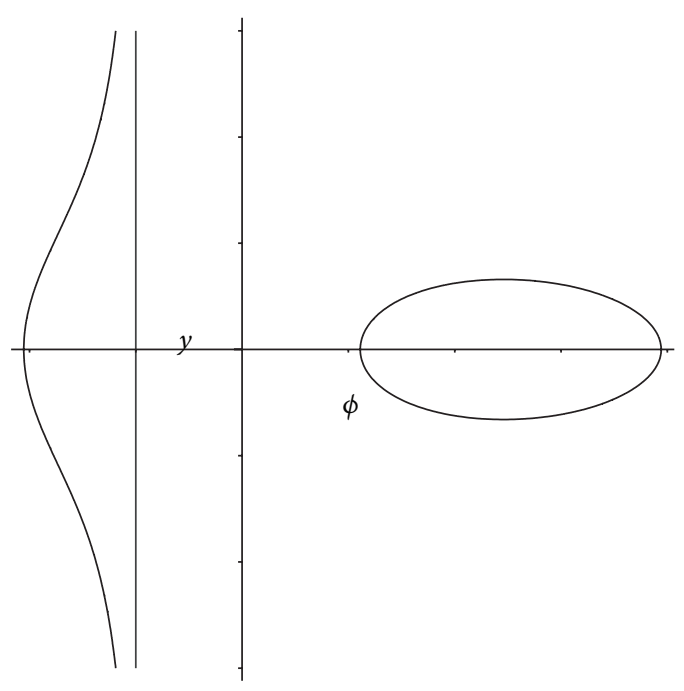

(d)

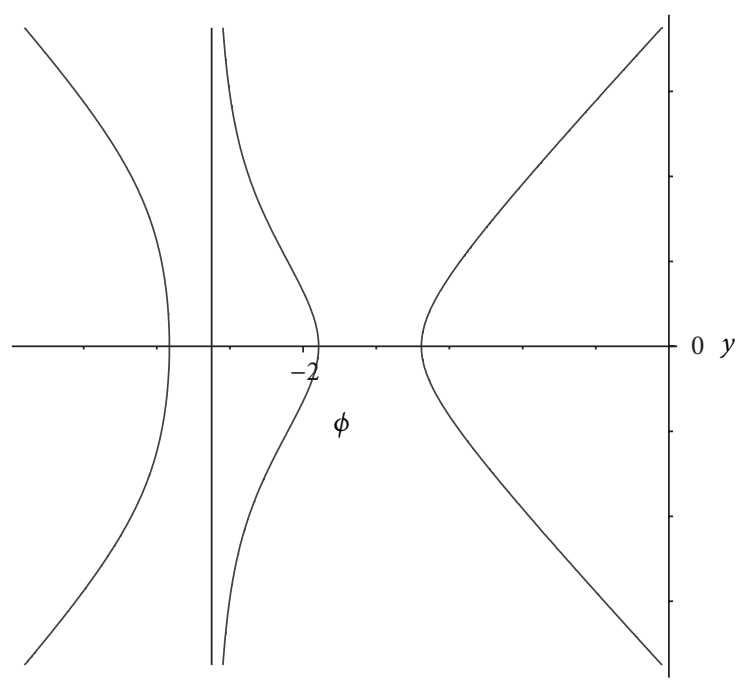

(f)

FIgure 6: Continued. 


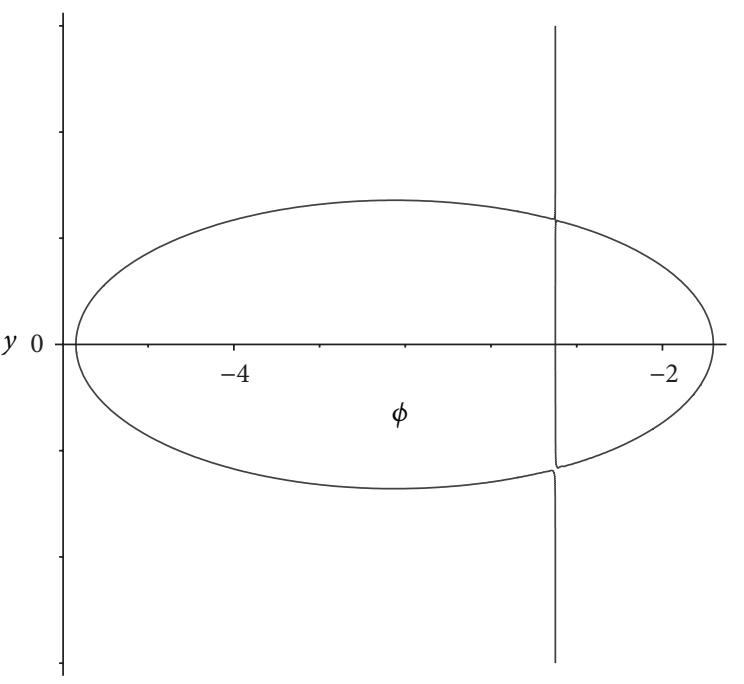

(g)

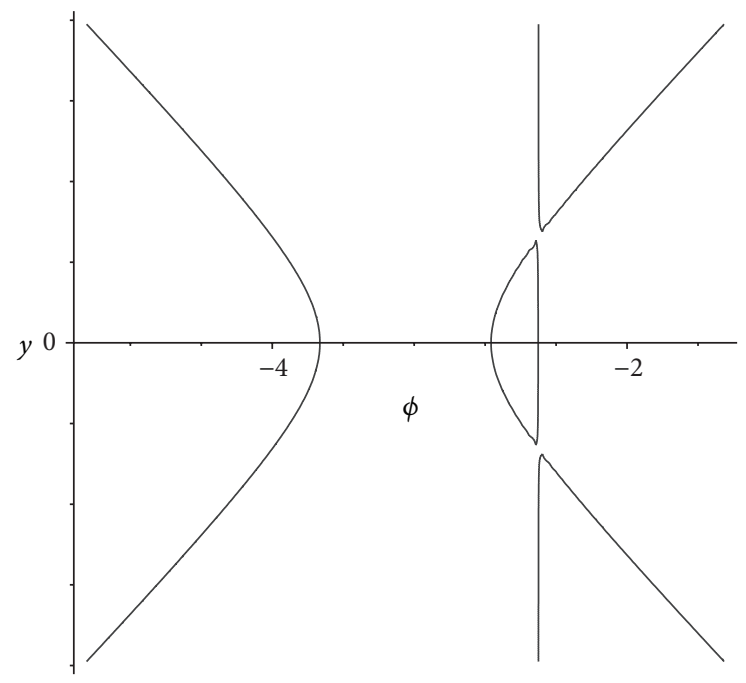

(i)

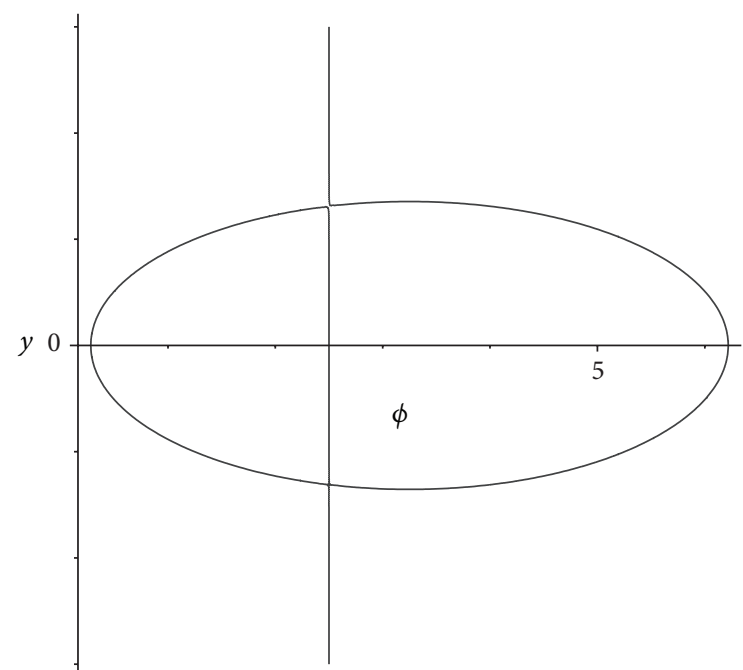

(h)

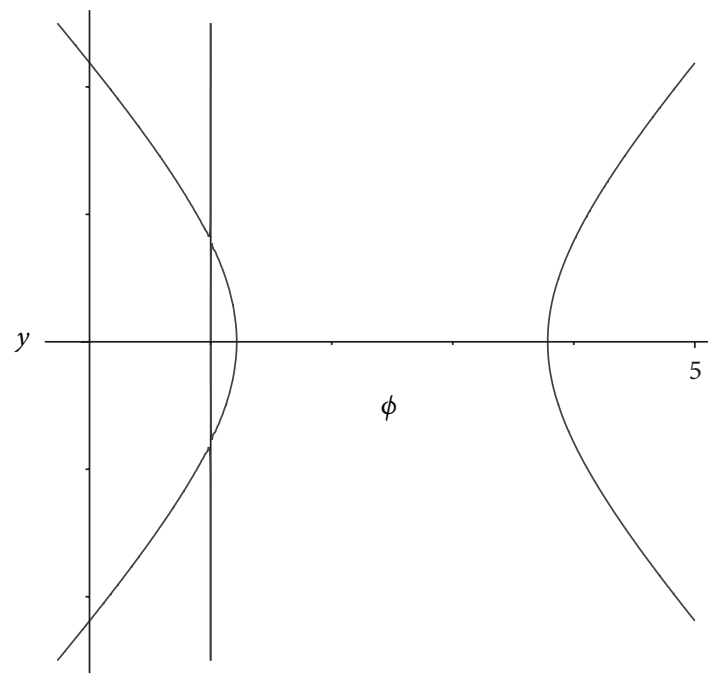

(j)

FIgURE 6: The level curves defined by $H(\phi, y)=h$.

Completing (34) and using transformation (4), we can get the implicit representation of a compacton solution of (3) as follows:

$$
\begin{aligned}
\frac{1}{\alpha_{1}^{2}}(\widetilde{u} & \left.+\left(\alpha_{1}^{2}-1\right) \Pi\left(\widetilde{\phi}, \alpha_{1}^{2}, k\right)\right) \\
& =\frac{\sqrt{\left(\phi_{s}-\gamma_{2}\right)\left(\gamma_{1}-\gamma_{3}\right)}}{2\left(\phi_{s}-\gamma_{1}\right) \sqrt{6 \alpha}}|x-c t|,
\end{aligned}
$$

where $\widetilde{u}=\operatorname{sn}^{-1}\left(\sqrt{\left(\phi_{s}-\gamma_{2}\right)\left(u(x, t)-\gamma_{1}\right) /\left(\phi_{s}-\gamma_{1}\right)\left(u(x, t)-\gamma_{2}\right)}\right.$, $k), \tilde{\phi}=\sin ^{-1}\left(\sqrt{\left(\phi_{s}-\gamma_{2}\right)\left(u(x, t)-\gamma_{1}\right) /\left(\phi_{s}-\gamma_{1}\right)\left(u(x, t)-\gamma_{2}\right)}\right)$, $k=\sqrt{\left(\phi_{s}-\gamma_{1}\right)\left(\gamma_{2}-\gamma_{3}\right) /\left(\phi_{s}-\gamma_{2}\right)\left(\gamma_{1}-\gamma_{3}\right)}$, and $\alpha_{1}^{2}=\left(\phi_{s}-\right.$ $\left.\gamma_{1}\right) /\left(\phi_{s}-\gamma_{2}\right) ; \Pi(\cdot, \cdot, \cdot)$ is Legendre's incomplete elliptic integral of the third kind, and $\mathrm{sn}^{-1}(\cdot, \cdot)$ is the inverse function of the Jacobian elliptic function $\operatorname{sn}(\cdot, \cdot)[15]$.
From Figure 6(b), we see that there are a periodic orbit and an open curve of system (7) defined by $H(\phi, y)=h$ if and only if one of the following conditions holds:

(i) $\alpha>0, c<-\beta / 2 \alpha,-c^{2} / 2<g<\beta(4 \alpha c+\beta) / 8 \alpha^{2}$, $h \in\left(h_{s}, h_{2}\right)$, and $h_{1}<h_{s}$,

(ii) $\alpha>0, c<-\beta / 2 \alpha, g=\beta(4 \alpha c+\beta) / 8 \alpha^{2}$, and $h \in$ $\left(h_{1}, h_{2}\right)$,

(iii) $\alpha>0, c \neq-\beta / 2 \alpha, g>\beta(4 \alpha c+\beta) / 8 \alpha^{2}$, and $h \in\left(h_{s}, h_{2}\right)$.

The open curve passes point $\left(\gamma_{1}, 0\right)$ and approaches the line $\phi=\phi_{s}$; its expression is

$$
y= \pm \frac{1}{\sqrt{6 \alpha}} \sqrt{\frac{\left(\gamma_{1}-\phi\right)\left(\phi-\gamma_{2}\right)\left(\phi-\gamma_{3}\right)}{\phi-\phi_{s}}}, \quad \phi_{s}<\phi \leq \gamma_{1} \text {, }
$$

where $\gamma_{1}, \gamma_{2}$, and $\gamma_{3}\left(\gamma_{3}<\gamma_{2}<\phi_{s}<\gamma_{1}\right)$ are three real roots of $\psi^{3}-3 c \psi^{2}-6 g \psi-3 h=0$. For example, $\gamma_{1} \approx 0.6863083159$, 
$\gamma_{2} \approx 0.243331752$, and $\gamma_{3} \approx-0.1796400679$ when $\alpha=1, \beta=$ $-1, c=0.25, g=0$, and $h=-0.01$ and $\gamma_{1} \approx 5.467670461, \gamma_{2} \approx$ $-1.060802034, \gamma_{3} \approx-1.406868427$ when $\alpha=1, \beta=2, c=1$, $g=2$, and $h=2.72$.

Substituting (36) into $d \phi / d \xi=y$ and integrating it along the open curve yield equation

$$
\int_{\phi}^{\gamma_{1}} \sqrt{\frac{s-\phi_{s}}{\left(\gamma_{1}-s\right)\left(s-\gamma_{2}\right)\left(s-\gamma_{3}\right)}} d s=\frac{1}{\sqrt{6 \alpha}} \int_{0}^{\xi} d s .
$$

Completing (37) and using transformation (4), we can get the implicit representation of a compacton solution of (3) as follows:

$$
\begin{aligned}
\frac{1}{\alpha_{1}^{2}}(\tilde{u} & \left.+\left(\alpha_{1}^{2}-1\right) \Pi\left(\tilde{\phi}, \alpha_{1}^{2}, k\right)\right) \\
& =\frac{\sqrt{\left(\gamma_{1}-\gamma_{2}\right)\left(\phi_{s}-\gamma_{3}\right)}}{2\left(\gamma_{1}-\phi_{s}\right) \sqrt{6 \alpha}}|x-c t|,
\end{aligned}
$$

where $\tilde{u}=\operatorname{sn}^{-1}\left(\sqrt{\left(\phi_{s}-\gamma_{3}\right)\left(\gamma_{1}-u(x, t)\right) /\left(\gamma_{1}-\phi_{s}\right)\left(u(x, t)-\gamma_{3}\right)}\right.$, $k), \tilde{\phi}=\sin ^{-1}\left(\sqrt{\left(\phi_{s}-\gamma_{3}\right)\left(\gamma_{1}-u(x, t)\right) /\left(\gamma_{1}-\phi_{s}\right)\left(u(x, t)-\gamma_{3}\right)}\right)$, $k=\sqrt{\left(\gamma_{1}-\phi_{s}\right)\left(\gamma_{2}-\gamma_{3}\right) /\left(\gamma_{1}-\gamma_{2}\right)\left(\phi_{s}-\gamma_{3}\right)}$, and $\alpha_{1}^{2}=\left(\phi_{s}-\right.$ $\left.\gamma_{1}\right) /\left(\phi_{s}-\gamma_{3}\right)$.

From Figure 6(c), we see that there are a periodic orbit and an open curve of system (7) defined by $H(\phi, y)=h$ when $\alpha>0, c>-\beta / 2 \alpha,-c^{2} / 2<g<\beta(4 \alpha c+\beta) / 8 \alpha^{2}, h \in\left(h_{s}, h_{2}\right)$, $h_{s}>h_{1}\left(\right.$ or $h \in\left(h_{1}, h_{2}\right)$, and $\left.h_{s}<h_{1}\right)$. The open curve passes point $\left(\gamma_{3}, 0\right)$ and approaches the line $\phi=\phi_{s}$; its expression is

$$
y= \pm \frac{1}{\sqrt{6 \alpha}} \sqrt{\frac{\left(\gamma_{1}-\phi\right)\left(\gamma_{2}-\phi\right)\left(\gamma_{3}-\phi\right)}{\phi-\phi_{s}}}, \quad \phi_{s}<\phi \leq \gamma_{3}
$$

where $\gamma_{1}, \gamma_{2}$, and $\gamma_{3}\left(\phi_{s}<\gamma_{3}<\gamma_{2}<\gamma_{1}\right)$ are three real roots of $\psi^{3}-3 c \psi^{2}-6 g \psi-3 h=0$. For example, $\gamma_{1} \approx-1.725343175$, $\gamma_{2} \approx-1.954509591$, and $\gamma_{3} \approx-2.320147234$ when $\alpha=0.2$, $\beta=1, c=-2, g=-1.985$, and $h=-2.608$ and $\gamma_{1} \approx$ $0.9464101615, \gamma_{2}=0.3$, and $\gamma_{3} \approx 0.2535898385$ when $\alpha=2$, $\beta=-1, c=0.5, g=-0.1$, and $h=0.024$.

Substituting (39) into $d \phi / d \xi=y$ and integrating it along the open curve yield equation

$$
\int_{\phi}^{\gamma_{3}} \sqrt{\frac{s-\phi_{s}}{\left(\gamma_{1}-s\right)\left(\gamma_{2}-s\right)\left(\gamma_{3}-s\right)}} d s=\frac{1}{\sqrt{6 \alpha}} \int_{0}^{\xi} d s .
$$

Completing (40) and using transformation (4), we can get the implicit representation of a compacton solution of (3) as follow:

$$
\begin{aligned}
\frac{1}{\alpha_{1}^{2}}(\tilde{u} & \left.+\left(\alpha_{1}^{2}-1\right) \Pi\left(\tilde{\phi}, \alpha_{1}^{2}, k\right)\right) \\
& =\frac{\sqrt{\left(\gamma_{1}-\gamma_{3}\right)\left(\gamma_{2}-\phi_{s}\right)}}{2\left(\gamma_{3}-\phi_{s}\right) \sqrt{6 \alpha}}|x-c t|,
\end{aligned}
$$

where $\tilde{u}=\operatorname{sn}^{-1}\left(\sqrt{\left(\gamma_{2}-\phi_{s}\right)\left(\gamma_{3}-u(x, t)\right) /\left(\gamma_{3}-\phi_{s}\right)\left(\gamma_{2}-u(x, t)\right)}\right.$, k), $\tilde{\phi}=\sin ^{-1}\left(\sqrt{\left(\gamma_{2}-\phi_{s}\right)\left(\gamma_{3}-u(x, t)\right) /\left(\gamma_{3}-\phi_{s}\right)\left(\gamma_{2}-u(x, t)\right)}\right)$, $k=\sqrt{\left(\gamma_{1}-\gamma_{2}\right)\left(\gamma_{3}-\phi_{s}\right) /\left(\gamma_{1}-\gamma_{3}\right)\left(\gamma_{2}-\phi_{s}\right)}$, and $\alpha_{1}^{2}=\left(\gamma_{3}-\right.$ $\left.\phi_{s}\right) /\left(\gamma_{2}-\phi_{s}\right)$.

From Figure 6(d), we see that there are a periodic orbit and an open curve of system (7) defined by $H(\phi, y)=h$ if and only if one of the following conditions holds:

(i) $\alpha>0, c>-\beta / 2 \alpha,-c^{2} / 2<g<\beta(4 \alpha c+\beta) / 8 \alpha^{2}$, $h \in\left(h_{1}, h_{s}\right)$, and $h_{s}<h_{2}$,

(ii) $\alpha>0, c>-\beta / 2 \alpha, g=\beta(4 \alpha c+\beta) / 8 \alpha^{2}$, and $h \epsilon$ $\left(h_{1}, h_{2}\right)$,

(iii) $\alpha>0, c \neq-\beta / 2 \alpha, g>\beta(4 \alpha c+\beta) / 8 \alpha^{2}$, and $h \in\left(h_{1}, h_{s}\right)$.

The open curve passes point $\left(\gamma_{3}, 0\right)$ and approaches the line $\phi=\phi_{s}$, its expression is

$$
y= \pm \frac{1}{\sqrt{6 \alpha}} \sqrt{\frac{\left(\gamma_{1}-\phi\right)\left(\gamma_{2}-\phi\right)\left(\phi-\gamma_{3}\right)}{\phi_{s}-\phi}}, \quad \gamma_{3} \leq \phi<\phi_{s},
$$

where $\gamma_{1}, \gamma_{2}$, and $\gamma_{3}\left(\gamma_{3}<\phi_{s}<\gamma_{2}<\gamma_{1}\right)$ are three real roots of $\psi^{3}-3 c \psi^{2}-6 g \psi-3 h=0$. For example, $\gamma_{1} \approx 2.557900553$, $\gamma_{2} \approx 0.7351513251$, and $\gamma_{3} \approx-2.393051878$ when $\alpha=1$, $\beta=-1, c=0.3, g=1$, and $h=-1.5$ and $\gamma_{1} \approx 5.743824982$, $\gamma_{2} \approx-0.6449004894, \gamma_{3} \approx-8.098924493$ when $\alpha=0.1, \beta=$ $1, c=-1, g=7.5$, and $h=10$.

Substituting (42) into $d \phi / d \xi=y$ and integrating it along the open curve yield equation

$$
\int_{\gamma_{3}}^{\phi} \sqrt{\frac{\phi_{s}-s}{\left(\gamma_{1}-s\right)\left(\gamma_{2}-s\right)\left(s-\gamma_{3}\right)}} d s=\frac{1}{\sqrt{6 \alpha}} \int_{0}^{\xi} d s .
$$

Completing (43) and using transformation (4), we can get the implicit representation of a compacton solution of (3) as follows:

$$
\begin{aligned}
\frac{1}{\alpha_{1}^{2}}(\tilde{u} & \left.+\left(\alpha_{1}^{2}-1\right) \Pi\left(\tilde{\phi}, \alpha_{1}^{2}, k\right)\right) \\
& =\frac{\sqrt{\left(\gamma_{1}-\phi_{s}\right)\left(\gamma_{2}-\gamma_{3}\right)}}{2\left(\phi_{s}-\gamma_{3}\right) \sqrt{6 \alpha}}|x-c t|,
\end{aligned}
$$

where $\tilde{u}=\operatorname{sn}^{-1}\left(\sqrt{\left(\gamma_{1}-\phi_{s}\right)\left(u(x, t)-\gamma_{3}\right) /\left(\phi_{s}-\gamma_{3}\right)\left(\gamma_{1}-u(x, t)\right)}\right.$, k), $\tilde{\phi}=\sin ^{-1}\left(\sqrt{\left(\gamma_{1}-\phi_{s}\right)\left(u(x, t)-\gamma_{3}\right) /\left(\phi_{s}-\gamma_{3}\right)\left(\gamma_{1}-u(x, t)\right)}\right)$, $k=\sqrt{\left(\gamma_{1}-\gamma_{2}\right)\left(\phi_{s}-\gamma_{3}\right) /\left(\gamma_{1}-\phi_{s}\right)\left(\gamma_{2}-\gamma_{3}\right)}$, and $\alpha_{1}^{2}=\left(\gamma_{3}-\right.$ $\left.\phi_{s}\right) /\left(\gamma_{1}-\phi_{s}\right)$.

From Figure 6(e), we see that there are three open curves of system (7) defined by $H(\phi, y)=h$ if and only if one of the following conditions holds:

(i) $\alpha<0, c<-\beta / 2 \alpha, g>-\left(12 \alpha^{2} c^{2}-4 \alpha \beta c-\beta^{2}\right) / 32 \alpha^{2}$, and $h \in\left(h_{s}, h_{2}\right)$,

(ii) $\alpha<0, c>-\beta / 2 \alpha, g>\beta(4 \alpha c+\beta) / 8 \alpha^{2}$, and $h \epsilon$ $\left(h_{s}, h_{2}\right)$.

One of them passes point $\left(\gamma_{2}, 0\right)$ and approaches the line $\phi=$ $\phi_{s}$; its expression is

$$
y= \pm \frac{1}{\sqrt{-6 \alpha}} \sqrt{\frac{\left(\gamma_{1}-\phi\right)\left(\phi-\gamma_{2}\right)\left(\phi-\gamma_{3}\right)}{\phi_{s}-\phi}}, \quad \gamma_{2} \leq \phi<\phi_{s},
$$


where $\gamma_{1}, \gamma_{2}$, and $\gamma_{3}\left(\gamma_{3}<\gamma_{2}<\phi_{s}<\gamma_{1}\right)$ are three real roots of $\psi^{3}-3 c \psi^{2}-6 g \psi-3 h=0$. For example, $\gamma_{1} \approx 2.064177772, \gamma_{2} \approx$ -0.3054072893 , and $\gamma_{3} \approx-4.758770483$ when $\alpha=-2, \beta=2$, $c=-1$, and $g=1.5$ and $h=1, \gamma_{1} \approx 5.466333036, \gamma_{2} \approx$ -1.035930847 , and $\gamma_{3} \approx-1.430402188$ when $\alpha=-0.5, \beta=$ $-1, c=1, g=2$, and $h=2.7$.

Substituting (45) into $d \phi / d \xi=y$ and integrating it along the open curve yield equation

$$
\int_{\gamma_{2}}^{\phi} \sqrt{\frac{\phi_{s}-s}{\left(\gamma_{1}-s\right)\left(s-\gamma_{2}\right)\left(s-\gamma_{3}\right)}} d s=\frac{1}{\sqrt{-6 \alpha}} \int_{0}^{\xi} d s
$$

Completing (46) and using transformation (4), we can get the implicit representation of a compacton solution of (3) as follows:

$$
\begin{aligned}
\frac{1}{\alpha_{1}^{2}}(\tilde{u} & \left.+\left(\alpha_{1}^{2}-1\right) \Pi\left(\tilde{\phi}, \alpha_{1}^{2}, k\right)\right) \\
& =\frac{\sqrt{\left(\gamma_{1}-\gamma_{2}\right)\left(\phi_{s}-\gamma_{3}\right)}}{2\left(\phi_{s}-\gamma_{2}\right) \sqrt{-6 \alpha}}|x-c t|,
\end{aligned}
$$

where $\tilde{u}=\operatorname{sn}^{-1}\left(\sqrt{\left(\phi_{s}-\gamma_{3}\right)\left(u(x, t)-\gamma_{2}\right) /\left(\phi_{s}-\gamma_{2}\right)\left(u(x, t)-\gamma_{3}\right)}\right.$, $k), \tilde{\phi}=\sin ^{-1}\left(\sqrt{\left(\phi_{s}-\gamma_{3}\right)\left(u(x, t)-\gamma_{2}\right) /\left(\phi_{s}-\gamma_{2}\right)\left(u(x, t)-\gamma_{3}\right)}\right)$, $k=\sqrt{\left(\phi_{s}-\gamma_{2}\right)\left(\gamma_{1}-\gamma_{3}\right) /\left(\gamma_{1}-\gamma_{2}\right)\left(\phi_{s}-\gamma_{3}\right)}$, and $\alpha_{1}^{2}=\left(\phi_{s}-\right.$ $\left.\gamma_{2}\right) /\left(\phi_{s}-\gamma_{3}\right)$.

From Figure 6(f), we see that there are three open curves of system (7) defined by $H(\phi, y)=h$ if and only if one of the following conditions holds:

(i) $\alpha<0, c<-\beta / 2 \alpha, g>\beta(4 \alpha c+\beta) / 8 \alpha^{2}$, and $h \in$ $\left(h_{1}, h_{s}\right)$,

(ii) $\alpha\langle 0, c\rangle-\beta / 2 \alpha, g\rangle-\left(12 \alpha^{2} c^{2}-4 \alpha \beta c-\beta^{2}\right) / 32 \alpha^{2}$, and $h \in\left(h_{1}, h_{s}\right)$.

One of them passes point $\left(\gamma_{2}, 0\right)$ and approaches the line $\phi=$ $\phi_{s}$; its expression is

$$
y= \pm \frac{1}{\sqrt{-6 \alpha}} \sqrt{\frac{\left(\gamma_{1}-\phi\right)\left(\gamma_{2}-\phi\right)\left(\phi-\gamma_{3}\right)}{\phi-\phi_{s}}}, \quad \phi_{s}<\phi \leq \gamma_{2}
$$

where $\gamma_{1}, \gamma_{2}$, and $\gamma_{3}\left(\gamma_{3}<\phi_{s}<\gamma_{2}<\gamma_{1}\right)$ are three real roots of $\psi^{3}-3 c \psi^{2}-6 g \psi-3 h=0$. For example, $\gamma_{1} \approx 0.8657007565$, $\gamma_{2} \approx-0.091826066674$, and $\gamma_{3} \approx-3.77387469$ when $\alpha=-2$, $\beta=-1, c=-1, g=0.5$, and $h=0.1$, and $\gamma_{1} \approx 6.154523009$, $\gamma_{2} \approx 2.662391044$, and $\gamma_{3} \approx 0.1830859473$ when $\alpha=-0.2$, $\beta=1, c=3, g=-3$, and $h=1$.

Substituting (48) into $d \phi / d \xi=y$ and integrating it along the open curve yield equation

$$
\int_{\phi}^{\gamma_{2}} \sqrt{\frac{s-\phi_{s}}{\left(\gamma_{1}-s\right)\left(\gamma_{2}-s\right)\left(s-\gamma_{3}\right)}} d s=\frac{1}{\sqrt{-6 \alpha}} \int_{0}^{\xi} d s .
$$

Completing (49) and using transformation (4), we can get the implicit representation of a compacton solution of (3) as follows:

$$
\begin{aligned}
\frac{1}{\alpha_{1}^{2}}(\tilde{u} & \left.+\left(\alpha_{1}^{2}-1\right) \Pi\left(\widetilde{\phi}, \alpha_{1}^{2}, k\right)\right) \\
& =\frac{\sqrt{\left(\gamma_{1}-\phi_{s}\right)\left(\gamma_{2}-\gamma_{3}\right)}}{2\left(\gamma_{2}-\phi_{s}\right) \sqrt{-6 \alpha}}|x-c t|,
\end{aligned}
$$

where $\tilde{u}=\operatorname{sn}^{-1}\left(\sqrt{\left(\gamma_{1}-\phi_{s}\right)\left(\gamma_{2}-u(x, t)\right) /\left(\gamma_{2}-\phi_{s}\right)\left(\gamma_{1}-u(x, t)\right)}\right.$, $k), \tilde{\phi}=\sin ^{-1}\left(\sqrt{\left(\gamma_{1}-\phi_{s}\right)\left(\gamma_{2}-u(x, t)\right) /\left(\gamma_{2}-\phi_{s}\right)\left(\gamma_{1}-u(x, t)\right)}\right)$, $k=\sqrt{\left(\gamma_{2}-\phi_{s}\right)\left(\gamma_{1}-\gamma_{3}\right) /\left(\gamma_{1}-\phi_{s}\right)\left(\gamma_{2}-\gamma_{3}\right)}$, and $\alpha_{1}^{2}=\left(\gamma_{2}-\right.$ $\left.\phi_{s}\right) /\left(\gamma_{1}-\phi_{s}\right)$.

3.5. Periodic Cusp Wave Solutions. For given $h=h_{s}$ in Figure 1(e), the level curve defined by $H(\phi, y)=h$ is shown in Figure 6(g).

For given $h=h_{s}$ in Figure $1(\mathrm{j})$, the level curve defined by $H(\phi, y)=h$ is shown in Figure 6(h).

For given $h=h_{s}$ in Figure 2(e), the level curve defined by $H(\phi, y)=h$ is shown in Figure 6(i).

For given $h=h_{s}$ in Figure 2(l), the level curve defined by $H(\phi, y)=h$ is shown in Figure $6(\mathrm{j})$.

From Figures 6(g) and 6(h), we see that there are two heteroclinic orbits of system (7) defined by $H(\phi, y)=h_{s}$ connecting with the saddle points $\left(\phi_{s}, Y_{ \pm}\right)$and passing points $\left(\phi_{m}, 0\right)$, and $\left(\phi_{M}, 0\right)$, respectively, when $\alpha>0, c \neq-\beta / 2 \alpha$, and $g>\beta(4 \alpha c+\beta) / 8 \alpha^{2}$, where $\phi_{M, m}=((\beta+6 \alpha c) \pm \sqrt{\delta}) / 4 \alpha$ and $\delta=-3 \beta^{2}-12 \alpha \beta c+36 \alpha^{2} c^{2}+96 \alpha^{2} g$. Their expressions are, respectively,

$$
\begin{array}{ll}
y= \pm \frac{1}{\sqrt{6 \alpha}} \sqrt{\left(\phi-\phi_{m}\right)\left(\phi_{M}-\phi\right)}, & \phi_{m} \leq \phi \leq \phi_{s}, \\
y= \pm \frac{1}{\sqrt{6 \alpha}} \sqrt{\left(\phi-\phi_{m}\right)\left(\phi_{M}-\phi\right),} \quad \phi_{s} \leq \phi \leq \phi_{M} .
\end{array}
$$

Substituting (51) into the $d \phi / d \xi=y$ and integrating it along the heteroclinic orbit, yields equation

$$
\int_{\phi}^{\phi_{s}} \frac{d s}{\sqrt{\left(s-\phi_{m}\right)\left(\phi_{M}-s\right)}}=\frac{1}{\sqrt{6 \alpha}} \int_{0}^{\xi} d s
$$

Completing (53) and using transformation (4), we can get a periodic cusp wave solution of (3) as follows:

$$
\begin{aligned}
u(x, t)= & \phi_{m} \cos ^{2}(\Omega-\omega|x-c t-2 n T|) \\
& +\phi_{M} \sin ^{2}(\Omega-\omega|x-c t-2 n T|), \\
& x-c t \in[(2 n-1) T,(2 n+1) T],
\end{aligned}
$$

where $n=0, \pm 1, \pm 2, \ldots, \omega=1 / 2 \sqrt{6 \alpha}, \Omega=$ $\tan ^{-1}\left(\sqrt{\left(\phi_{s}-\phi_{m}\right) /\left(\phi_{M}-\phi_{s}\right)}\right)$, and $T=2|\Omega|$.

Substituting (52) into $d \phi / d \xi=y$ and integrating it along the heteroclinic orbit yield equation

$$
\int_{\phi_{s}}^{\phi} \frac{d s}{\sqrt{\left(s-\phi_{m}\right)\left(\phi_{M}-s\right)}}=\frac{1}{\sqrt{6 \alpha}} \int_{0}^{\xi} d s .
$$


Completing (55) and using transformation (4), we can get a periodic cusp wave solution of (3) as follows:

$$
\begin{aligned}
u(x, t)= & \phi_{m} \sin ^{2}(\Omega+\omega|x-c t-2 n T|) \\
& +\phi_{M} \cos ^{2}(\Omega+\omega|x-c t-2 n T|), \\
& x-c t \in[(2 n-1) T,(2 n+1) T],
\end{aligned}
$$

where $n=0, \pm 1, \pm 2, \ldots, \omega=1 / 2 \sqrt{6 \alpha}, \Omega=$ $\tan ^{-1}\left(\sqrt{\left(\phi_{M}-\phi_{s}\right) /\left(\phi_{s}-\phi_{m}\right)}\right)$, and $T=2|\Omega|$.

From Figure 6(i), we see that there are a heteroclinic orbit and an open curve of system (7) defined by $H(\phi, y)=h_{s}$ when $\alpha<0, c<-\beta / 2 \alpha$, and $-\left(12 \alpha^{2} c^{2}-4 \alpha \beta c-\beta^{2}\right) / 32 \alpha^{2}<g<$ $\beta(4 \alpha c+\beta) / 8 \alpha^{2}$. The heteroclinic orbit connecting with the saddle points $\left(\phi_{s}, Y_{ \pm}\right)$and passing point $\left(\phi_{M}, 0\right)$; its expression is

$$
y= \pm \frac{1}{\sqrt{-6 \alpha}} \sqrt{\left(\phi-\phi_{M}\right)\left(\phi-\phi_{m}\right)}, \quad \phi_{M} \leq \phi \leq \phi_{s}
$$

where $\phi_{m, M}=((\beta+6 \alpha c) \pm \sqrt{\delta}) / 4 \alpha$ and $\delta=-3 \beta^{2}-12 \alpha \beta c+$ $36 \alpha^{2} c^{2}+96 \alpha^{2} g$.

Substituting (57) into $d \phi / d \xi=y$ and integrating it along the heteroclinic orbit yield equation

$$
\int_{\phi}^{\phi_{s}} \frac{d s}{\sqrt{\left(s-\phi_{M}\right)\left(s-\phi_{m}\right)}}=\frac{1}{\sqrt{-6 \alpha}} \int_{0}^{\xi} d s .
$$

Completing (58) and using transformation (4), we can get a periodic cusp wave solution of (3) as follows:

$$
\begin{aligned}
u(x, t)= & \phi_{M} \cosh ^{2}(\Omega-\omega|x-c t-2 n T|) \\
& -\phi_{m} \sinh ^{2}(\Omega-\omega|x-c t-2 n T|), \\
& x-c t \in[(2 n-1) T,(2 n+1) T],
\end{aligned}
$$

where $n=0, \pm 1, \pm 2, \ldots, \omega=1 / 2 \sqrt{-6 \alpha}, \Omega=$ $\tanh ^{-1}\left(\sqrt{\left(\phi_{s}-\phi_{M}\right) /\left(\phi_{s}-\phi_{m}\right)}\right)$, and $T=2|\Omega|$.

From Figure $6(\mathrm{j})$, we see that there are a heteroclinic orbit and an open curve of system (7) defined by $H(\phi, y)=h_{s}$ when $\alpha<0, c>-\beta / 2 \alpha$, and $-\left(12 \alpha^{2} c^{2}-4 \alpha \beta c-\beta^{2}\right) / 32 \alpha^{2}<g<$ $\beta(4 \alpha c+\beta) / 8 \alpha^{2}$. The heteroclinic orbit connecting with the saddle points $\left(\phi_{s}, Y_{ \pm}\right)$and passing point $\left(\phi_{m}, 0\right)$; its expression is

$$
y= \pm \frac{1}{\sqrt{-6 \alpha}} \sqrt{\left(\phi_{m}-\phi\right)\left(\phi_{M}-\phi\right)}, \quad \phi_{s} \leq \phi \leq \phi_{m}
$$

where $\phi_{m, M}=((\beta+6 \alpha c) \pm \sqrt{\delta}) / 4 \alpha$ and $\delta=-3 \beta^{2}-12 \alpha \beta c+$ $36 \alpha^{2} c^{2}+96 \alpha^{2} g$.

Substituting (60) into $d \phi / d \xi=y$ and integrating it along the heteroclinic orbit yield equation

$$
\int_{\phi_{s}}^{\phi} \frac{d s}{\sqrt{\left(\phi_{m}-s\right)\left(\phi_{M}-s\right)}}=\frac{1}{\sqrt{-6 \alpha}} \int_{0}^{\xi} d s .
$$

Completing (61) and using transformation (4), we can get a periodic cusp wave solution of (3) as follows:

$$
\begin{aligned}
u(x, t)= & \phi_{m} \cosh ^{2}(\Omega+\omega|x-c t-2 n T|) \\
& -\phi_{M} \sinh ^{2}(\Omega+\omega|x-c t-2 n T|), \\
& x-c t \in[(2 n-1) T,(2 n+1) T],
\end{aligned}
$$

where $n=0, \pm 1, \pm 2, \ldots, \omega=1 / 2 \sqrt{-6 \alpha}, \Omega=$ $\tanh ^{-1}\left(\sqrt{\left(\phi_{m}-\phi_{s}\right) /\left(\phi_{M}-\phi_{s}\right)}\right)$, and $T=2|\Omega|$.

\section{Numerical Simulations}

In this section, we simulate the planar diagrams of the compactons and the periodic cusp waves of (3). From the derivations of (6) and (7), it is seen that if $\phi=\phi(\xi)$ and $y=y(\xi)$ are the parameter expressions of an orbit of system (7) and they satisfy $\phi(0)=\phi_{0}$ and $y(0)=y_{0}$, then the diagram of $u=\phi(\xi)$ and the integral curve of (6) with initial conditions $\phi(0)=\phi_{0}$ and $\phi^{\prime}(0)=y_{0}$ are the same. Therefore we can use the simulations of integral curves of (6) to test the validity of exact travelling wave solutions (35), (38), (41), (44), (47), (50), (54), (56), (59), and (62).

Example 1. Choose $\alpha, \beta, c, g$, and $h$ satisfying the parametric conditions of Figure 6(a); that is $\alpha=0.2, \beta=1, c=$ $-3, g=-4.48$, and $h=-8.88$, and let $\phi(0)=-2.6$ in (33), then it follows that $\phi^{\prime}(0)=y(0) \approx 0.3651483603$ or $\phi^{\prime}(0)=y(0) \approx-0.3651483603$. Taking $\phi(0)=-2.6$, and $y(0)=0.3651483603$ as initial values of (6), using the Maple, we get the numerical simulation of the integral curve which corresponds to the open curve (33) as Figure 7(a).

Example 2. Choose $\alpha, \beta, c, g$, and $h$ satisfying the parametric conditions of Figure 6(b); that is $\alpha=0.2, \beta=0, c=-1$, $g=-0.25$, and $h=0.2$, and let $\phi(0)=0.001$ in (36); then it follows that $\phi^{\prime}(0)=y(0) \approx 22.33265544$ or $\phi^{\prime}(0)=y(0) \approx$ -22.33265544 . Taking $\phi(0)=0.001$, and $y(0)=22.33265544$ as initial values of (6), using the Maple, we get the numerical simulation of the integral curve which corresponds to the open curve (36) as Figure 7(b).

Example 3. Choose $\alpha, \beta, c, g$, and $h$ satisfying the parametric conditions of Figure 6(c); that is $\alpha=0.2, \beta=1, c=-2$, $g=-1.985, h=-2.608$ and let $\phi(0)=-2.4$ in (39); then it follows that $\phi^{\prime}(0)=y(0) \approx 0.4472135955$ or $\phi^{\prime}(0)=$ $y(0) \approx-0.4472135955$. Taking $\phi(0)=-2.4$ and $y(0)=$ -0.4472135955 as initial values of (6), using the Maple, we get the numerical simulation of the integral curve which corresponds to the open curve (39) as Figure 7(c).

Example 4. Choose $\alpha, \beta, c, g$, and $h$ satisfying the parametric conditions of Figure $6(\mathrm{~d})$; that is $\alpha=0.2, \beta=0, c=2$, $g=0$, and $h=-5$, and let $\phi(0)=-1.2$ in (42); then it follows that $\phi^{\prime}(0)=y(0) \approx 1.793506807$ or $\phi^{\prime}(0)=y(0) \approx$ -1.793506807 . Taking $\phi(0)=-1.2$ and $y(0)=1.793506807$ as initial values of (6), using the Maple, we get the numerical simulation of the integral curve which corresponds to the open curve (42) as Figure 7(d). 


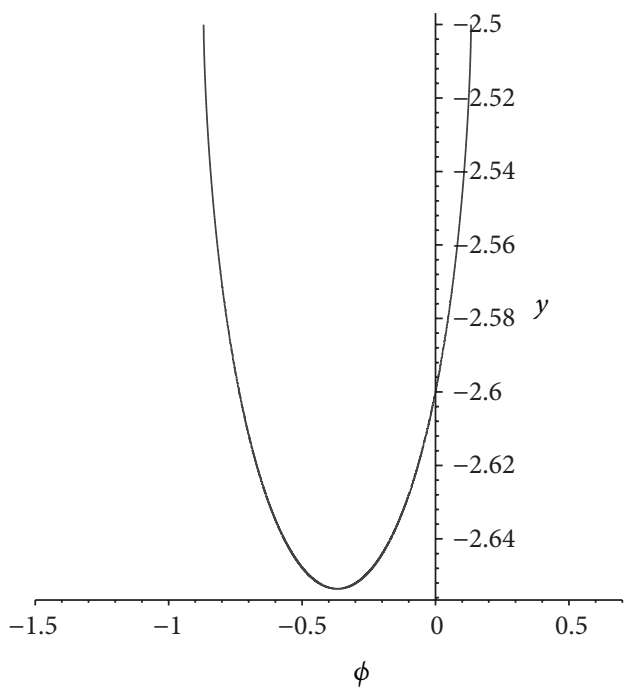

(a)

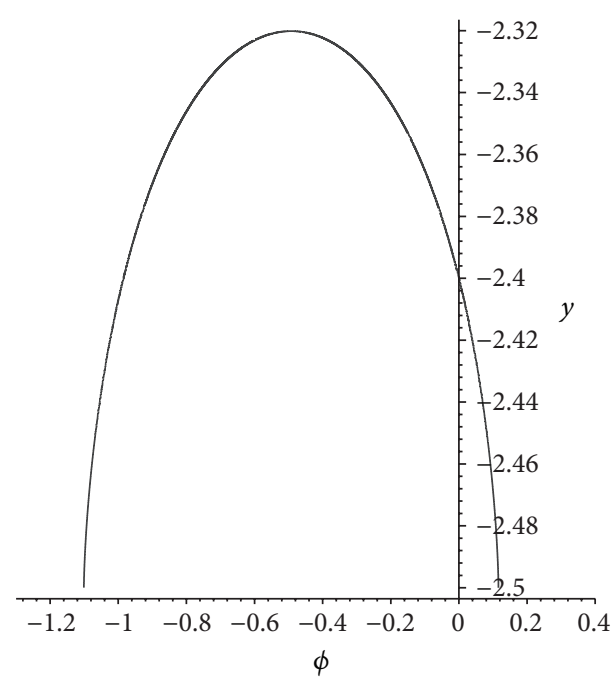

(c)

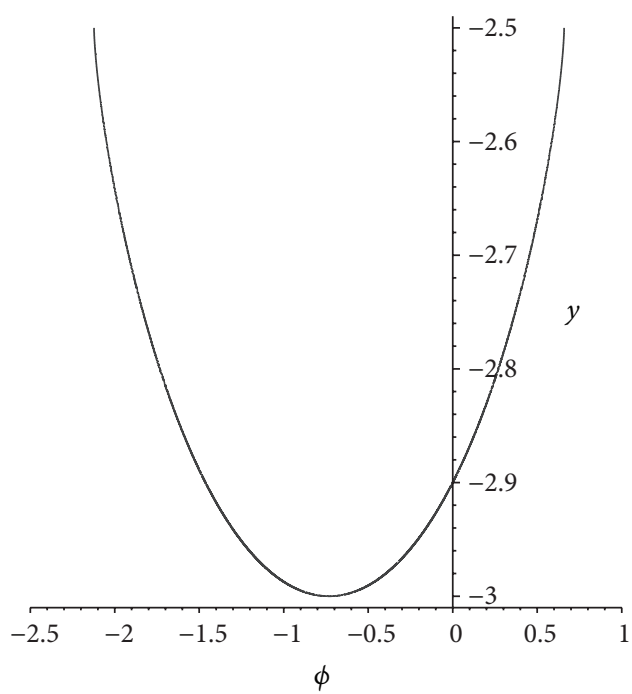

(e)

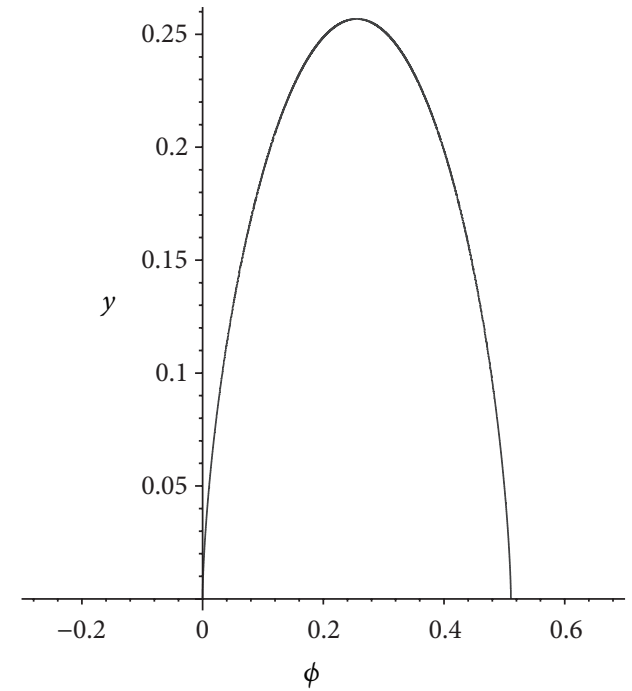

(b)

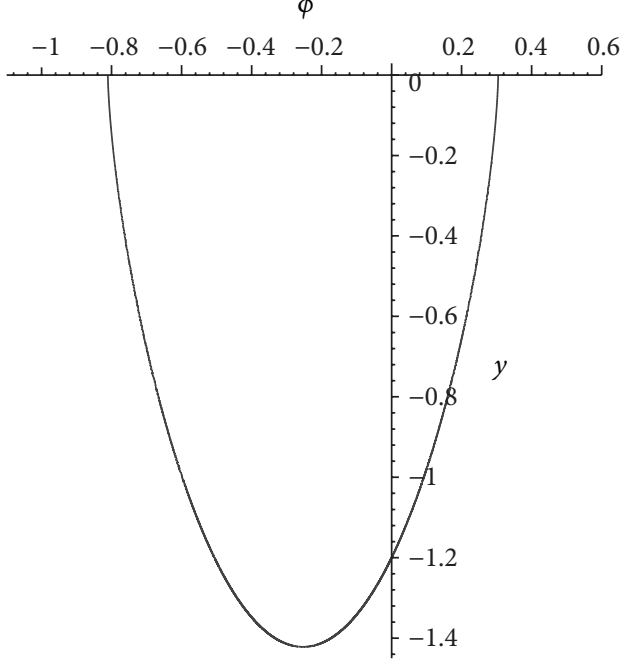

(d)

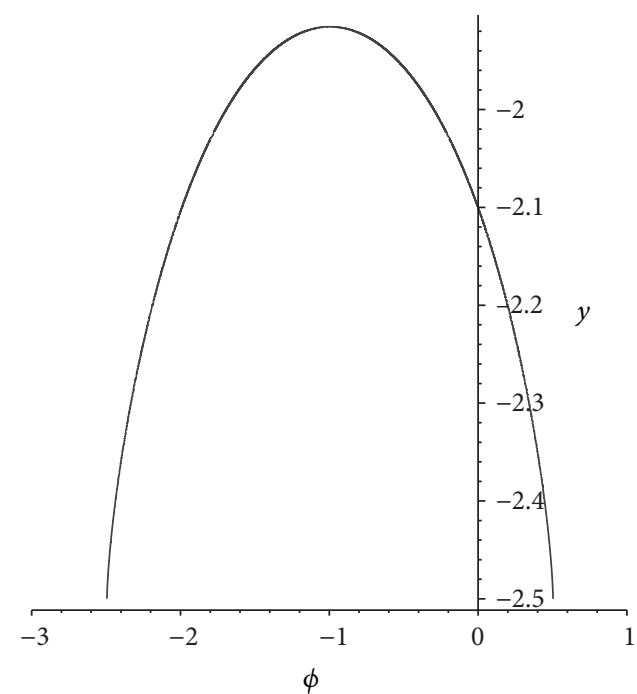

(f)

FIgURE 7: Continued. 


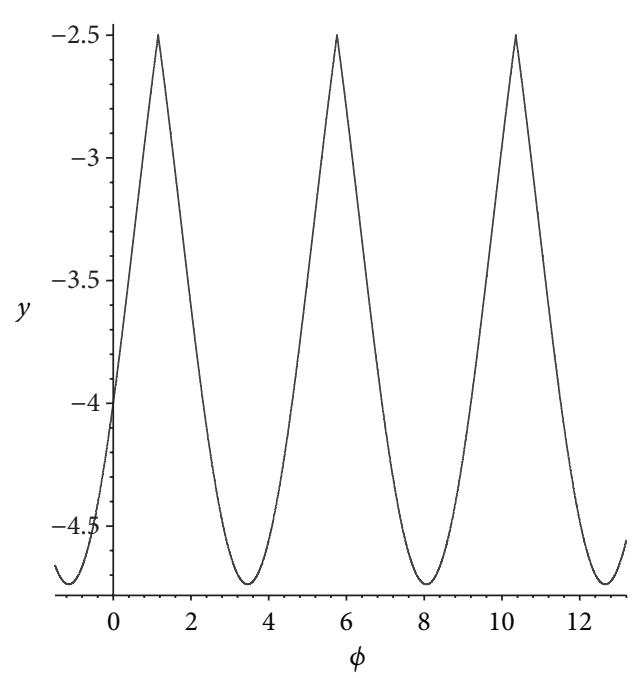

(g)

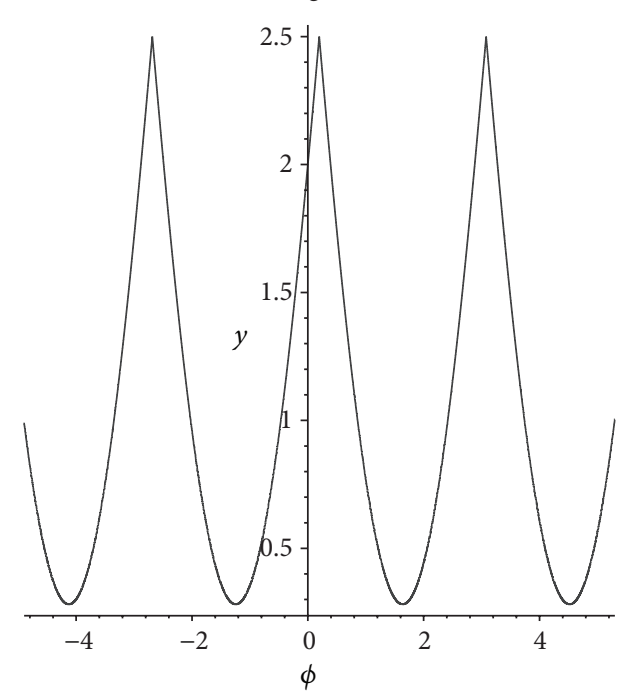

(i)

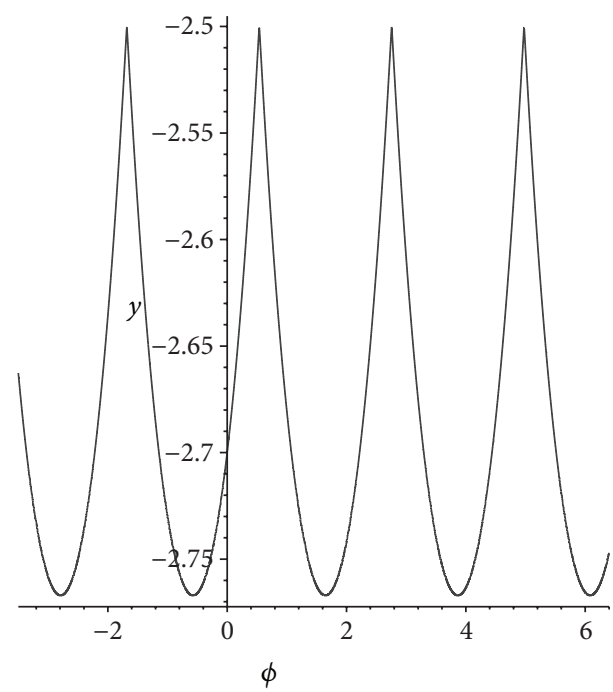

(k)

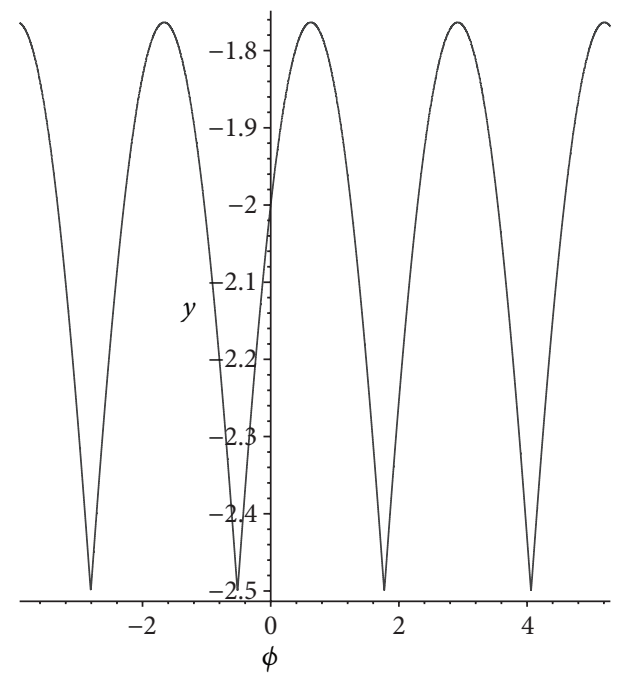

(h)

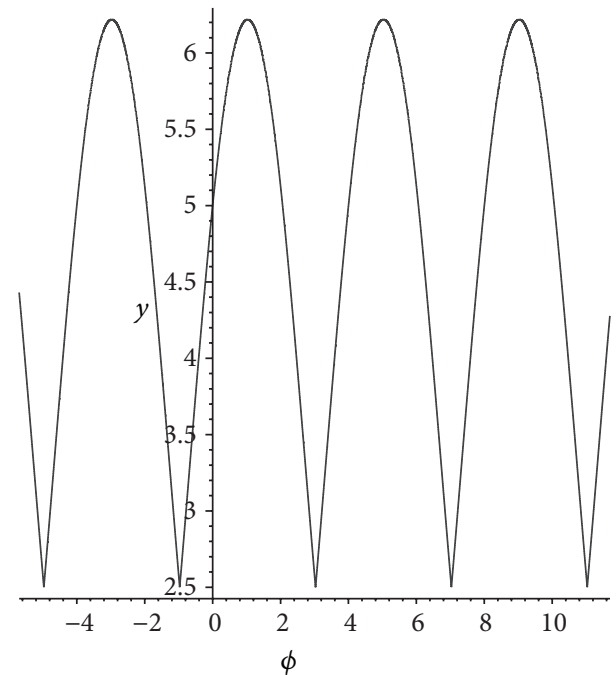

(j)

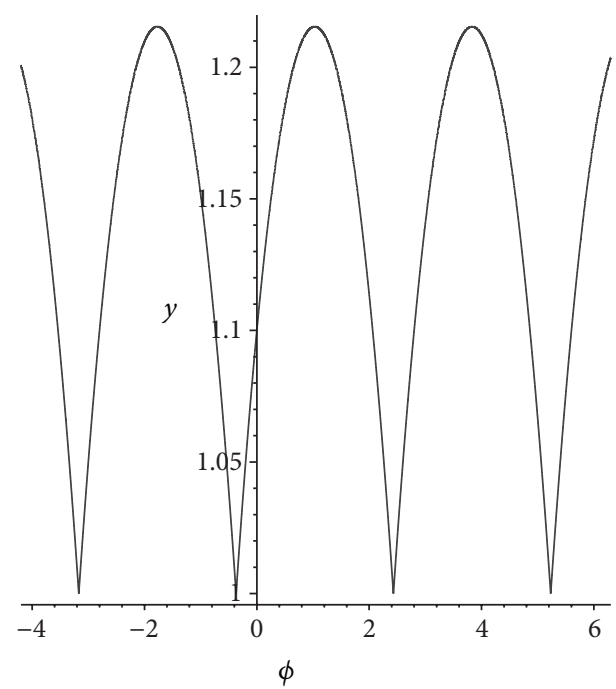

(l)

FIgURE 7: The numerical simulations of integral curves of (3). 
Example 5. Choose $\alpha, \beta, c, g$, and $h$ satisfying the parametric conditions of Figure 6(e); that is $\alpha=-0.2, \beta=-1, c=-3$, $g=-4.43, h=-8.58$, and let $\phi(0)=-2.9$ in $(45)$; then it follows that $\phi^{\prime}(0)=y(0) \approx 0.2922613022$ or $\phi^{\prime}(0)=$ $y(0) \approx-0.2922613022$. Taking $\phi(0)=-2.9$ and $y(0)=$ 0.2922613022 as initial values of (6), using the Maple, we get the numerical simulation of the integral curve which corresponds to the open curve (45) as Figure 7(e).

Example 6. Choose $\alpha, \beta, c, g$, and $h$ satisfying the parametric conditions of Figure $6(\mathrm{f})$; that is $\alpha=-0.2, \beta=-1, c=$ $-2, g=-1.92$, and $h=-2.36$, and let $\phi(0)=-2.1$ in (48); then it follows that $\phi^{\prime}(0)=y(0) \approx 0.4257346591$ or $\phi^{\prime}(0)=y(0) \approx-0.4257346591$. Taking $\phi(0)=-2.1$ and $y(0)=0.4257346591$ as initial values of (6), using the Maple, we get the numerical simulation of the integral curve which corresponds to the open curve (48) as Figure 7(f).

Example 7. Choose $\alpha, \beta, c$, and $g$ satisfying the parametric conditions of Figure $6(\mathrm{~g})$; that is $\alpha=0.2, \beta=1, c=-3$, and $g=-4$.1. Let $\phi(0)=-4$ in (51); then it follows that $\phi^{\prime}(0)=$ $y(0) \approx 1.172603942$ or $\phi^{\prime}(0)=y(0) \approx-1.172603942$. Taking $\phi(0)=-4$ and $y(0)=1.172603942$ as initial values of (6), using the Maple, we get the numerical simulation of the integral curve which corresponds to the heteroclinic orbit (51) as Figure $7(\mathrm{~g})$. Let $\phi(0)=-2$ in (52), then it follows that $\phi^{\prime}(0)=y(0) \approx 0.7337120246$ or $\phi^{\prime}(0)=y(0) \approx$ -0.7337120246 . Taking $\phi(0)=-2, y(0)=0.7337120246$ as initial values of (6), using the Maple, we get the numerical simulation of the integral curve which corresponds to the heteroclinic orbit (52) as Figure 7(h).

Example 8. Choose $\alpha, \beta, c$, and $g$ satisfying the parametric conditions of Figure $6(\mathrm{~h})$; that is $\alpha=0.2, \beta=-1, c=3$, and $g=-3$. Let $\phi(0)=2$ in (51), then it follows that $\phi^{\prime}(0)=$ $y(0) \approx 2.457912394$ or $\phi^{\prime}(0)=y(0) \approx-2.457912394$. Taking $\phi(0)=2$ and $y(0)=2.457912394$ as initial values of (6), using the Maple, we get the numerical simulation of the integral curve which corresponds to the heteroclinic orbit (51) as Figure 7(i). Let $\phi(0)=5$ in (52); then it follows that $\phi^{\prime}(0)=$ $y(0) \approx 2.188957133$ or $\phi^{\prime}(0)=y(0) \approx-2.188957133$. Taking $\phi(0)=5, y(0)=2.188957133$ as initial values of (6), using the Maple, we get the numerical simulation of the integral curve which corresponds to the heteroclinic orbit (52) as Figure 7(j).

Example 9. Choose $\alpha, \beta, c$, and $g$ satisfying the parametric conditions of Figure 6(i); that is $\alpha=-0.2, \beta=-1, c=$ -3 , and $g=-4.43$ and let $\phi(0)=-2.7$ in (57), then it follows that $\phi^{\prime}(0)=y(0) \approx 0.2397915762$ or $\phi^{\prime}(0)=$ $y(0) \approx-0.2397915762$. Taking $\phi(0)=-2.7$ and $y(0)=$ 0.2397915762 as initial values of (6), using the Maple, we get the numerical simulation of the integral curve which corresponds to the heteroclinic orbit (57) as Figure 7(k).

Example 10. Choose $\alpha, \beta, c$, and $g$ satisfying the parametric conditions of Figure $6(\mathrm{j})$; that is $\alpha=-1, \beta=2, c=2$, and $g=$ -1.6 and let $\phi(0)=-1.1$ in $(60)$; then it follows that $\phi^{\prime}(0)=$ $y(0) \approx 0.2272296966$ or $\phi^{\prime}(0)=y(0) \approx-0.2272296966$. Taking $\phi(0)=1.1$ and $y(0)=0.2272296966$ as initial values of (6), using the Maple, we get the numerical simulation of the integral curve which corresponds to the heteroclinic orbit (60) as Figure 7(l).

Figure 7 implies that our theoretic results of the compacton and the periodic cusp wave solutions are identical with the numerical simulations.

\section{Conclusion}

In this paper, we studied the bifurcations of travelling wave solutions of (3) using the approach of dynamical system and obtained some exact peakon, compacton, solitary wave, smooth periodic wave, and periodic cusp wave solutions. Also, the compactons and the periodic cusp waves are simulated by approach of numerical simulation. The results of this paper have enriched results of references [9-11].

\section{Acknowledgments}

This work is supported by the Natural Science Foundation of Yunnan Province, China (no. 2013FZ117), and the National Natural Science Foundation of China (no. 11161020).

\section{References}

[1] P. Rosenau and J. M. Hyman, "Compactons: solitons with finite wavelength," Physical Review Letters, vol. 70, no. 5, pp. 564-567, 1993.

[2] J. DeFrutos, M. A. López-Marcos, and J. M. Sanz-Serna, "A finite difference scheme for the $K(2,2)$ compacton equation," Journal of Computational Physics, vol. 120, no. 2, pp. 248-252, 1995.

[3] M. S. Ismail and T. R. Taha, "A numerical study of compactons," Mathematics and Computers in Simulation, vol. 47, no. 6, pp. 519-530, 1998.

[4] A. M. Wazwaz, "New solitary-wave special solutions with compact support for the nonlinear dispersive $K(m, n)$ equations," Chaos, Solitons and Fractals, vol. 13, no. 2, pp. 321-330, 2002.

[5] J. Yin and L. Tian, "Classification of the travelling waves in the nonlinear dispersive KdV equation," Nonlinear Analysis. Theory, Methods \& Applications A, vol. 73, no. 2, pp. 465-470, 2010.

[6] B. Mihaila, A. Cardenas, F. Cooper, and A. Saxena, "Stability and dynamical properties of Rosenau-Hyman compactons using Padé approximants," Physical Review E, vol. 81, pp. 6708-6715, 2010.

[7] L. Zhang and J. Li, "Dynamical behavior of loop solutions for the $K(2,2)$ equation," Physics Letters A, vol. 375, no. 33, pp. 29652968, 2011.

[8] L. Zhang, A. Chen, and J. Tang, "Special exact soliton solutions for the $K(2,2)$ equation with non-zero constant pedestal," Applied Mathematics and Computation, vol. 218, no. 8, pp. 44484457, 2011.

[9] F. Cooper, H. Shepard, and P. Sodano, "Solitary waves in a class of generalized Korteweg-de Vries equations," Physical Review E, vol. 48, no. 5, pp. 4027-4032, 1993.

[10] B. Dey and A. Khare, "Stability of compacton solutions," Physical Review E, vol. 58, pp. 2741-2744, 1998.

[11] J. L. Yin, "Stability of smooth solitary waves for the generalized Korteweg-de-Vries equation with combined dispersion," 
Ukrainian Mathematical Journal, vol. 63, no. 8, pp. 1234-1240, 2012.

[12] J. Li and Z. Liu, "Smooth and non-smooth traveling waves in a nonlinearly dispersive equation," Applied Mathematical Modelling, vol. 25, no. 1, pp. 41-56, 2000.

[13] Z. Liu and C. Chen, "Compactons in a general compressible hyperelastic rod," Chaos, Solitons and Fractals, vol. 22, no. 3, pp. 627-640, 2004.

[14] B. He, "Bifurcations and exact bounded travelling wave solutions for a partial differential equation," Nonlinear Analysis. Real World Applications, vol. 11, no. 1, pp. 364-371, 2010.

[15] P. F. Byrd and M. D. Friedman, Handbook of Elliptic Integrals for Engineers and Scientists, Springer, Berlin, Germany, 1971. 


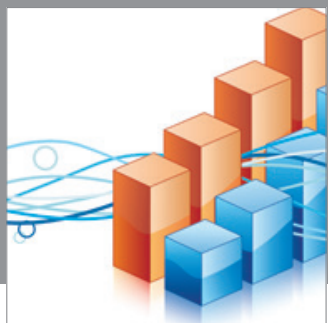

Advances in

Operations Research

mansans

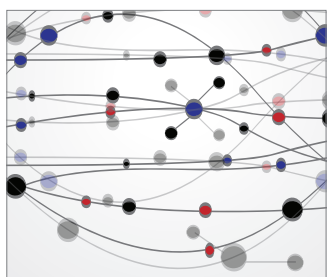

The Scientific World Journal
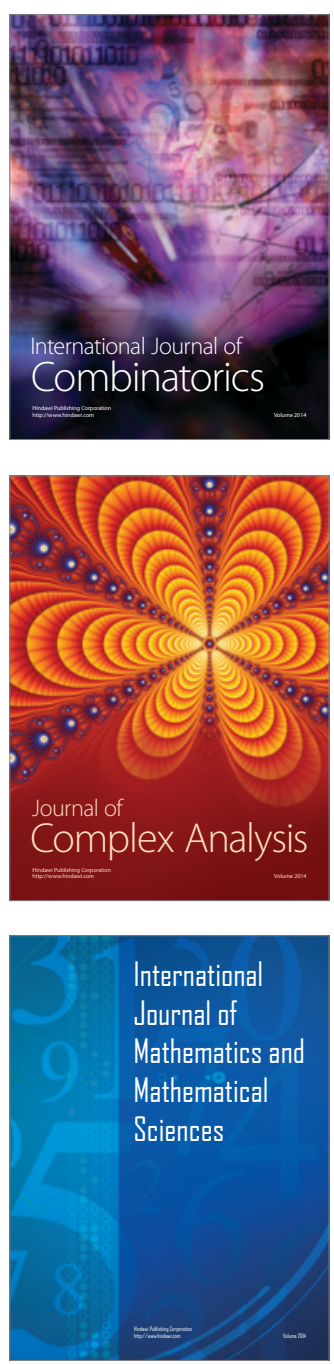
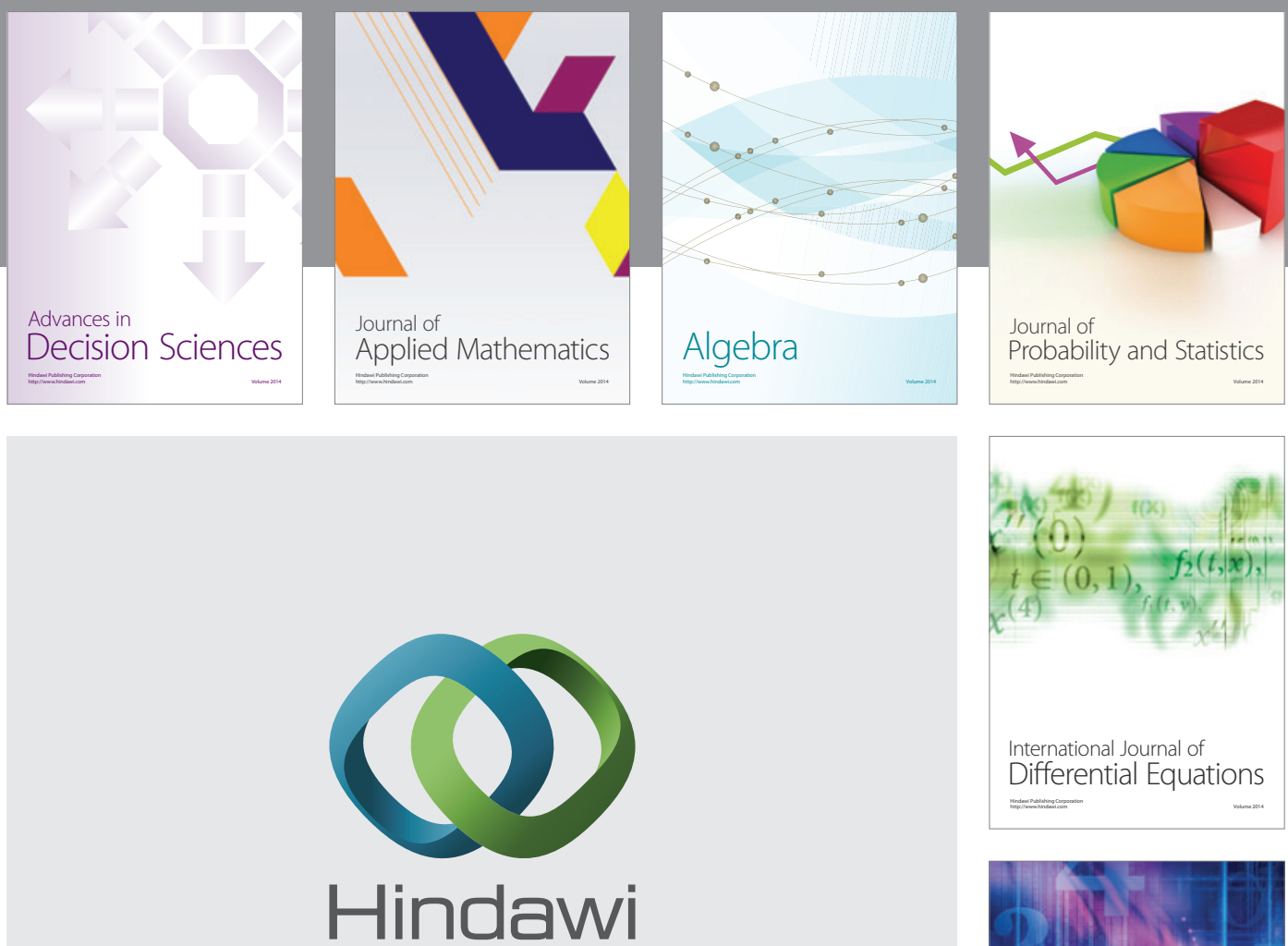

Submit your manuscripts at http://www.hindawi.com
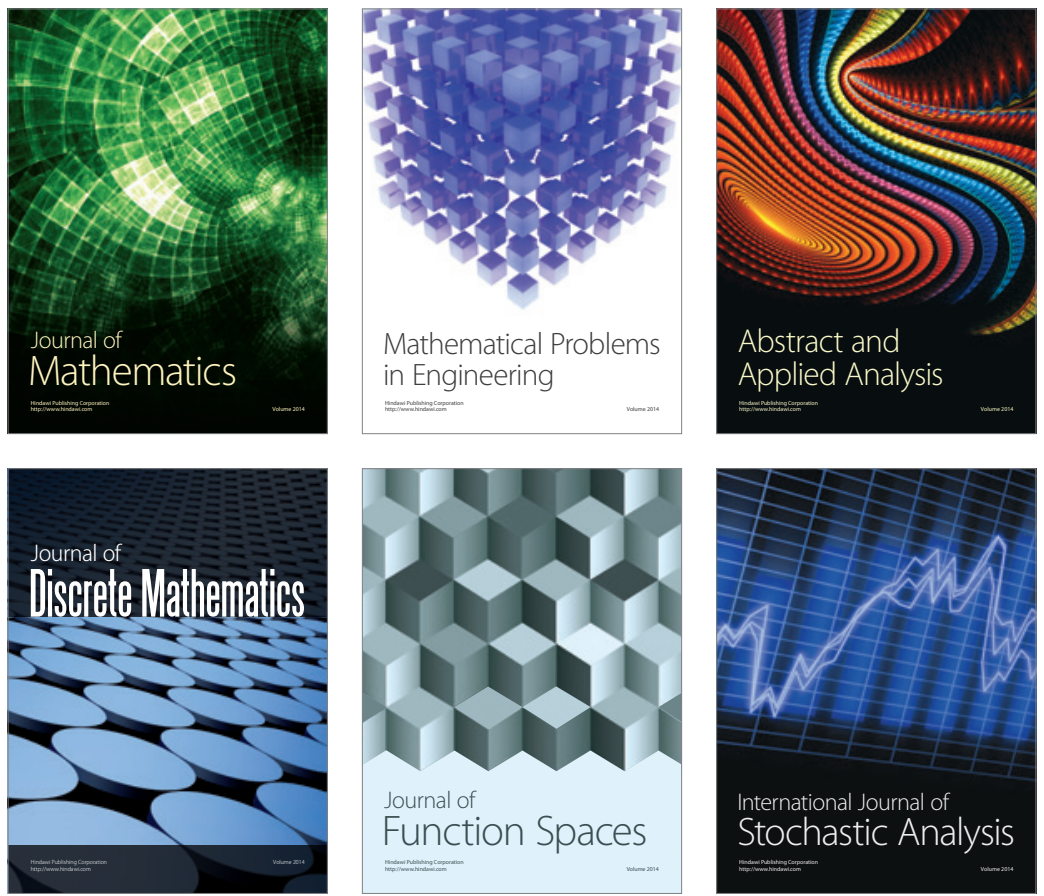

Journal of

Function Spaces

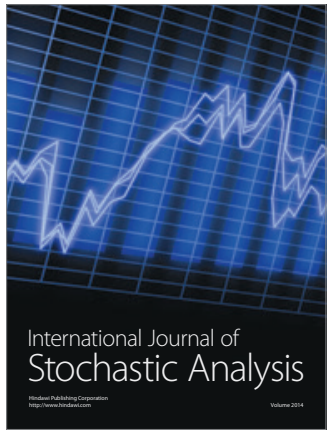

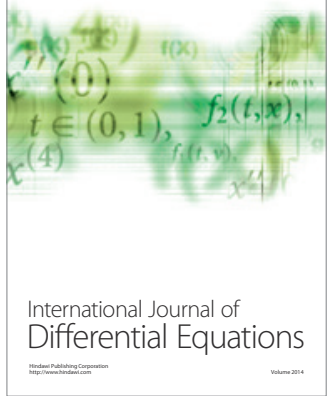
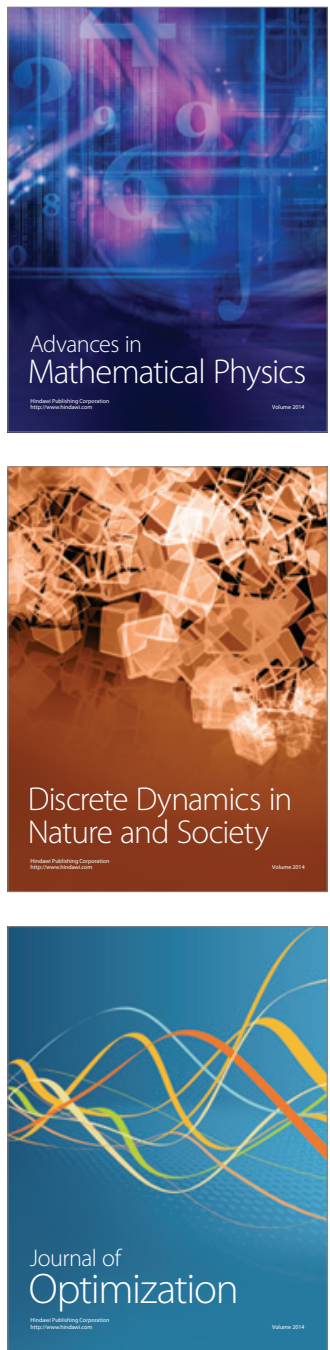